\title{
Galois coverings of one-sided bimodule problems
}

\author{
Vyacheslav Babych, Nataliya Golovashchuk
}

\begin{abstract}
Applying geometric methods of 2-dimensional cell complex theory, we construct a Galois covering of a one-sided bimodule problem satisfying some structure, triangularity and finiteness conditions. Each bimodule problem $\mathcal{A}$ from the considered class is endowed with a quasi multiplicative basis. The main result shows that for a finite dimensional problem from the considered class having schurian universal covering $\tilde{\mathcal{A}}$, either $\mathcal{A}$ is schurian, or its basic bigraph contains a dotted loop, or it has a standard minimal non-schurian bimodule subproblem.
\end{abstract}

Анотація. Застосовуючи геометричні методи теорії двовимірних клітинних комплексів, в роботі будується накриття Галуа односторонньої бімодульної задачі, яка задовольняє деякі умови структурності, трикутності та скінченності. Кожна бімодульна задача $\mathcal{A}$ з розглядуваного класу наділена квазімультиплікативним базисом. Основний результат показує, що для скінченновимірної задачі з розглядуваного класу, яка має шурівське універсальне накриття $\tilde{\mathcal{A}}$, або $\mathcal{A}$ сама $€$ шурівською, або їі базовий біграф містить пунктирну петлю, або вона містить стандартну мінімальну нешурівську бімодульну підзадачу.

2010 Mathematics Subject Classification: 57M20, 57M10, 16D90, 16P60, 15A04

Keywords: cell complex, covering, bimodule problem, Tits form, schurity

Ключові слова: клітинний комплекс, накриття, бімодульна задача, форма Тітса, шуровість

DOI: http://dx.doi.org/10.15673/tmgc.v14i2.1768 


\section{INTRODUCTION}

An idea to use geometric technique for solving a range of problems that arise naturally in algebra finds more and more applications $([5,9,16])$. In particular, geometric language has a number of advantages for a finiteness problem solution for various algebraic structures in representation theory [7, $10,11,15,19]$. These methods are similar to those used in geometric group theory ([18]), and include a useful tool called "covering method" ([8]) which is especially effective in the case when the basis of algebraic structure is multiplicative, i.e. the composition of two composable basic elements is either zero or a basic element too ([21]).

We apply covering technique to investigate representation category for a class of bimodule problems which are pairs consisting of a category and a bimodule over it ([1]). From the representation theory point of view, it is important to describe the classes of problems having a correspondence between the associated Tits quadratic form and the representation category. In the simplest cases (when the problems are schurian) the dimensions of indecomposable representations correspond to the positive roots of the Tits form $([13,17])$. For this reason, we need to determine the minimal non-schurian subproblems, and the proposed covering technique allows to solve this problem effectively for a considered class of so called one-sided bimodule problems (see section 3.3, [4]).

We introduced a quasi multiplicative basis generalizing the notion of a multiplicative one ([4]). Existence of such a basis makes it possible to apply geometric technique to investigation of bimodule problem representation category properties. Following [6], we define standard minimal non-schurian one-sided bimodule problem and use the result of [3] stating that minimal one-sided non-schurian bimodule problem with weakly positive Tits quadratic form is standard. A similar result was obtained by the authors for another class of bimodule problems in [2] previously.

We associate a 2-dimensional cell complex with a faithful one-sided bimodule problem in order to construct a corresponding Galois covering. In the case of a bimodule problem of finite type, a Galois covering induces a covering of corresponding representation category with the same fundamental group $([11,12])$. This fact allows to describe representation category of the initial bimodule problem using "simpler" representation category of the constructed Galois covering.

The main result (Theorem 4.7.1) states the existence of standard minimal non-schurian subproblem for a finite dimensional non-schurian bimodule problem having weakly positive associated Tits quadratic form and schurian universal covering. After $[3,4]$, this is the next step on the way 
of characterization of representation type for finite dimensional bimodule problems from the considered class.

\section{Preliminaries}

1.1. Quadratic forms. For a set $I$ and the set $\mathcal{P}_{I}$ of all its 2-element subsets, a polynomial

$$
q(x)=\sum_{i \in I} q_{i i} x_{i}^{2}+\sum_{\{i, j\} \in \mathcal{P}_{I}} q_{i j} x_{i} x_{j}
$$

in the variables $x_{i}$ with coefficients $q_{i j}=q_{j i} \in \mathbb{Z}, i, j \in I$, is called an integral quadratic form. If $q_{i i}=1$ for all $i \in I$, then $q(x)$ is called unit. We call $q$ locally finite whenever for every $i \in I$ there are finitely many $j \in I$ such that $q_{i j} \neq 0$. For an element $x=\left(x_{i}\right)_{i \in I} \in \mathbb{Z}^{I}$, the set

$$
\operatorname{supp} x=\left\{i \in I \mid x_{i} \neq 0\right\}
$$

is called the support of $x$. An element $x$ is called positive if $x_{i}>0$ for any $i \in \operatorname{supp} x$. We write $x>0$ in this case, and $x>y$ for $x, y \in \mathbb{Z}^{I}$ if $x-y>0$. An element $x>0$ with finite support satisfying condition $q(x)=1$ is called a (positive) root of the form $q$. The set of all positive roots of $q$ will be denoted by $\Re_{q}^{+}$. A basic element $e_{i}$ such that $\left(e_{i}\right)_{j}=\delta_{i j}, i, j \in I$, is called a simple root.

A quadratic form $q$ is called weakly positive or WP if $q(x)>0$ for any positive $x$ with finite support. Denote by WP the set of all weakly positive locally finite quadratic forms.

For $J \subset I$, define the restriction of a vector $x=\left(x_{i}\right)_{i \in I} \in \mathbb{Z}^{I}$ from $I$ to $J$ by the linear map

$$
\mathbb{Z}^{I} \ni\left(x_{i}\right)_{i \in I} \stackrel{\cdot J}{\longmapsto}\left(x_{i}\right)_{i \in J} \in \mathbb{Z}^{J}
$$

and an extension of a vector by 0 on $I$ by

$$
\mathbb{Z}^{J} \ni x \stackrel{. I}{\longmapsto} x^{I} \in \mathbb{Z}^{I}
$$

such that $\left(x^{I}\right)_{J}=x$ and $\left(x^{I}\right)_{I \backslash J}=0$. A restriction $q_{J}$ of a quadratic form $q$ on $J$ is defined by the equality

$$
q_{J}(x)=q\left(x^{I}\right), x \in \mathbb{Z}^{J} .
$$

The form $q_{J}$ is called a subform of the form $q$. If $J \neq I$, then the subform $q_{J}$ is called proper.

For a quadratic form $q$, we denote by $($,$) or (,)_{q}$ the corresponding symmetric bilinear form

$$
(x, y)=(q(x+y)-q(x)-q(y)) / 2 .
$$

Obviously,

$$
(x, x)=(q(2 x)-2 q(x)) / 2=q(x) .
$$


Let $q$ be integral form in finitely many variables, i.e. $|I|<\infty$. Then $q$ is WP if and only if $\left|\Re_{q}^{+}\right|<\infty$ ([20]). A root $x$ of $q$ is called sincere if $\operatorname{supp} x=I$. If $q$ has a sincere positive root, then $q$ is called sincere.

Lemma 1.1.1. Let $q\left(x_{1}, \ldots, x_{n}\right) \in \mathrm{WP}$ be an unit form, $n \geqslant 2, x \in \Re_{q}^{+}$ and $|\operatorname{supp} x|>1$. Then for any $i \in \operatorname{supp} x$ :

(1) $2\left(e_{i}, x\right) \in\{-1,0,1\}$;

(2) $x-2\left(e_{i}, x\right) e_{i} \in \Re_{q}^{+}$.

Proof. (1) We have that

$$
\begin{aligned}
& 2\left(e_{i}, x\right)=q\left(x+e_{i}\right)-q(x)-q\left(e_{i}\right)=q\left(x+e_{i}\right)-2>-2, \\
& 2\left(e_{i}, x\right)=q(x)+q\left(e_{i}\right)-q\left(x-e_{i}\right)=2-q\left(x-e_{i}\right)<2 .
\end{aligned}
$$

(2) Since $x-2\left(e_{i}, x\right) e_{i}>0$, we obtain

$$
q\left(x-2\left(e_{i}, x\right) e_{i}\right)=q(x)+q\left(2\left(e_{i}, x\right) e_{i}\right)-\left(2\left(e_{i}, x\right)\right)^{2}=q(x)=1 .
$$

1.2. Critical forms. A unit integral form $q\left(x_{1}, \ldots, x_{n}\right)$ with $n \geqslant 3$ variables is called critical if it is not WP, but every proper subform of $q$ is WP. The following facts are well known (see $[15,20]$ ).

- For a critical form $q$, there exists a minimal sincere positive integer vector $\mu$ (a critical vector) such that $q(\mu)=0$, and $q(y)>0$ for any positive $y<\mu$. Besides, $\left(\mu, e_{i}\right)=0$ and $q(\mu+z)=q(z)$ for every $i \in\{1, \ldots, n\}$ and $z \in \mathbb{Z}^{n}$. If $q(\nu)=0$ for $\nu>0$, then $\nu=k \mu$ for some positive integer $k$.

- If an unit integral quadratic form $q\left(x_{1}, \ldots, x_{n}\right)$ in $n \geqslant 3$ variables is not WP, then $q$ contains either a critical subform or a subform $x_{i}^{2}+x_{j}^{2}+$ $q_{i j} x_{i} x_{j}$, where $q_{i j} \leqslant-2,1 \leqslant i<j \leqslant n$.

Lemma 1.2.1. Let $q\left(x_{1}, \ldots, x_{n}\right)$ be a critical form, $\mu$ its critical vector, $y \in \Re_{q}^{+}$a sincere root such that $y<\mu$, and $i_{1}, i_{2} \in \operatorname{supp} y=\{1, \ldots, n\}=I$, $i_{1} \neq i_{2}$. Then the following statements hold.

1) $2\left(e_{i}, y\right) \in\{-2,-1,0,1\}$ for any $i \in I$.

2) There exists $j \in I$ such that $z=y-e_{j} \in \Re_{q}^{+}$and $i_{1}, i_{2} \in \operatorname{supp} z$.

3) There is non-sincere $x \in \Re_{q}^{+}$such that $x<y$ and $i_{1}, i_{2} \in \operatorname{supp} x$.

Proof. 1) Since $q\left(y+e_{i}\right)=0$ if $y+e_{i}=\mu$, and $q\left(y+e_{i}\right)>0$ if $y+e_{i}<\mu$ by definition of critical vector, we have

$$
2\left(e_{i}, y\right)=q\left(y+e_{i}\right)-q(y)-q\left(e_{i}\right)=q\left(y+e_{i}\right)-2 \geqslant-2 .
$$

Similarly, since $y-e_{i}>0$,

$$
2\left(e_{i}, y\right)=2-q\left(y-e_{i}\right)<2 .
$$


2) The equality

$$
2=2 q(y)=2(y, y)=\sum_{i=1}^{n} 2\left(e_{i}, y\right) y_{i}
$$

implies one of the statements: either

(1) there exists $j \neq i_{1}, i_{2}$ such that $2\left(e_{j}, y\right)=1$, or

(2) there is $j \in\left\{i_{1}, i_{2}\right\}$ such that $2\left(e_{j}, y\right)=1$ and $y_{j} \geqslant 2$, or

(3) $2\left(e_{i_{1}}, y\right)=2\left(e_{i_{2}}, y\right)=1, y_{i_{1}}=y_{i_{2}}=1$ and $\left(e_{j}, y\right)=0, j \in I \backslash\left\{i_{1}, i_{2}\right\}$.

In the first two cases the vector $z=y-e_{j}$ is a positive root of $q$ since $y$ is sincere and $q\left(y-e_{i}\right)=1$ and $i_{1}, i_{2} \in \operatorname{supp} z$. The third case is impossible since $(\mu, y)=0$ for a critical vector $\mu$, but, on the other hand,

$$
(\mu, y)=\sum_{i=1}^{n} \mu_{i}\left(e_{i}, y\right)=\mu_{i_{1}}\left(e_{i_{1}}, y\right)+\mu_{i_{2}}\left(e_{i_{2}}, y\right)=\left(\mu_{i_{1}}+\mu_{i_{2}}\right) / 2 \geqslant 1 .
$$

Statement 3) follows from 2).

1.3. Basic roots and singular vertices. Let $q\left(x_{1}, \ldots, x_{n}\right) \in \mathrm{WP}$ be an unit form, $n \geqslant 2$. A sincere $x \in \Re_{q}^{+}$is called a basic root, if there exist $i_{1}, i_{2} \in I=\{1, \ldots, n\}$ such that $2\left(e_{i_{1}}, x\right)=2\left(e_{i_{2}}, x\right)=1, x_{i_{1}}=x_{i_{2}}=1$ and $2\left(e_{i}, x\right)=0, i \in I \backslash\left\{i_{1}, i_{2}\right\}$. We will call $i_{1}$, $i_{2}$ the singular vertices of $x$.

Lemma 1.3.1 ([3, Lemma 1$])$. Let $q\left(x_{1}, \ldots, x_{n}\right) \in \mathrm{WP}$ be an unit form, $n \geqslant 3$, let $x \in \Re_{q}^{+}$be sincere non-basic root, and let $i_{1}, i_{2} \in I, i_{1} \neq i_{2}$. Then there exists non-sincere root $y \in \Re_{q}^{+}$such that $y<x$, and $i_{1}, i_{2} \in \operatorname{supp} y$.

\subsection{Bigraphs. A directed bigraph}

$$
\Sigma=\left(\Sigma_{0}, \Sigma_{1}, s, e, \operatorname{deg}\right)
$$

is given by a set $\Sigma_{0}$ of vertices, a set $\Sigma_{1}$ of arrows, two maps $s, e: \Sigma_{1} \rightarrow \Sigma_{0}$ defining an initial and a terminal vertices of an arrow, and the degree $\operatorname{deg}: \Sigma_{1} \rightarrow\{0,1\}$ indicating a type of an arrow. For $X, Y \in \Sigma_{0}$, let

$$
\Sigma_{1}(X, Y)=\left\{x \in \Sigma_{1} \mid s(x)=X, e(x)=Y\right\} .
$$

Here $\Sigma_{1}=\Sigma_{1}^{0} \sqcup \Sigma_{1}^{1}$, where $\Sigma_{1}^{i}=\operatorname{deg}^{-1}(i), i=0,1$. The arrows from $\Sigma_{1}^{0}$ $\left(\Sigma_{1}^{1}\right)$ are called solid (dotted). If

$$
\Sigma^{\prime}=\left(\Sigma_{0}^{\prime}, \Sigma_{1}^{\prime}, s^{\prime}, e^{\prime}, \operatorname{deg}^{\prime}\right)
$$

is another bigraph, then a morphism

$$
f: \Sigma \rightarrow \Sigma^{\prime}
$$

of bigraphs is a pair $f=\left(f_{0}, f_{1}\right)$, where $f_{0}: \Sigma_{0} \rightarrow \Sigma_{0}^{\prime}, f_{1}: \Sigma_{1} \rightarrow \Sigma_{1}^{\prime}$ such that $f_{0} s=s^{\prime} f_{1}, f_{0} e=e^{\prime} f_{1}$, and $\operatorname{deg}^{\prime} f_{1}=$ deg. A bigraph $\Sigma$ is called connected (0-connected) if there does not exist a partition $\Sigma_{0}=\Sigma_{0}^{1} \sqcup \Sigma_{0}^{2}$ into 
nonempty subsets such that $s(a) \in \Sigma_{0}^{i}$ implies $e(a) \in \Sigma_{0}^{i}$ for every $a \in \Sigma_{1}$ $\left(a \in \Sigma_{1}^{0}\right), i=1,2$. A connected bigraph is called:

- a loop, if $\left|\Sigma_{0}\right|=\left|\Sigma_{1}\right|=1$;

- a cycle $C_{n}$ if $\left|\Sigma_{0}\right|=\left|\Sigma_{1}\right|=n \geqslant 2$, and $\left|e^{-1}(A)\right|+\left|s^{-1}(A)\right|=2$ for any $A \in \Sigma_{0}$

- a chain, if there are $X_{1}, X_{2} \in \Sigma_{0}$ such that $\left|e^{-1}\left(X_{i}\right)\right|+\left|s^{-1}\left(X_{i}\right)\right|=1$, $i=1,2$, and $\left|e^{-1}(A)\right|+\left|s^{-1}(A)\right|=2$ for every $A \in \Sigma_{0} \backslash\left\{X_{1}, X_{2}\right\}$.

A bigraph $\Sigma$ is called locally finite if each $X \in \Sigma_{0}$ is incident to finitely many arrows, and finite provided $\Sigma_{0}$ and $\Sigma_{1}$ are finite.

1.5. Paths and walks in bigraph. For a bigraph

$$
\Sigma=\left(\Sigma_{0}, \Sigma_{1}, s, e, \operatorname{deg}\right)
$$

let $\Sigma_{1}^{-1}$ be the set of the elements $x^{-1}$ for all $x \in \Sigma_{1}$, and let $\hat{\Sigma}$ be the bigraph such that $\widehat{\Sigma}_{0}=\Sigma_{0}, \widehat{\Sigma}_{1}=\Sigma_{1} \sqcup \Sigma_{1}^{-1}$. The maps $s, e: \widehat{\Sigma}_{1} \rightarrow \widehat{\Sigma}_{0}$ and deg restricted on $\Sigma_{1} \subset \widehat{\Sigma}_{1}$ coincide with those for $\Sigma$, and

$$
e\left(x^{-1}\right)=s(x), s\left(x^{-1}\right)=e(x), \operatorname{deg} x^{-1}=\operatorname{deg} x .
$$

A walk (respectively a path) on $\Sigma$ of the length $m$ is a sequence

$$
\omega=x_{1} x_{2} \ldots x_{m}
$$

of arrows $x_{i} \in \widehat{\Sigma}_{1}$ (respectively $x_{i} \in \Sigma_{1}$ ), $1 \leqslant i \leqslant m$, such that

$$
s\left(x_{i}\right)=e\left(x_{i+1}\right)
$$

for all $1 \leqslant i<m$. We assume that

$$
s(\omega)=s\left(x_{m}\right), \quad e(\omega)=e\left(x_{1}\right),
$$

and for every $X \in \Sigma_{0}$ there exists a unique (trivial) path $\mathbb{1}_{X}$ of the length 0 such that $s\left(\mathbb{1}_{X}\right)=e\left(\mathbb{1}_{X}\right)=X$. The composition $\omega \omega^{\prime}$ of the walks $\omega$ and $\omega^{\prime}$ is naturally defined if $s(\omega)=e\left(\omega^{\prime}\right)$.

The walks and the paths on $\Sigma$ form the categories denoted by Walks $\Sigma$

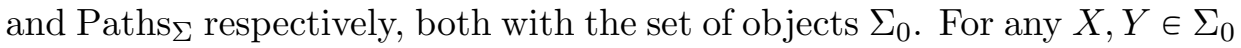
the set $\operatorname{Walks}_{\Sigma}(X, Y)\left(\operatorname{Paths}_{\Sigma}(X, Y)\right)$ contains all the walks (the paths) $\omega$ in $\Sigma$ such that $s(\omega)=X, e(\omega)=Y$. There exists an obvious inclusion functor

$$
\imath: \text { Paths }_{\Sigma} \hookrightarrow \text { Walks }_{\Sigma} .
$$

We denote by $W_{\Sigma}$ the groupoid of walks on $\Sigma$,

$$
\mathrm{W}_{\Sigma}=\mathrm{Walks}_{\Sigma} /\left(x \circ x^{-1}=\mathbb{1}_{e(x)}, x \in \widehat{\Sigma}_{1}\right),
$$

and by

$$
r_{\Sigma}: \mathrm{Walks}_{\Sigma} \rightarrow \mathrm{W}_{\Sigma}
$$

the canonical projection. 
A morphism

$$
f=\left(f_{0}, f_{1}\right): \Sigma \rightarrow \Sigma^{\prime}
$$

of bigraphs naturally induces a functor

$$
\text { Walks }_{f}: \text { Walks }_{\Sigma} \rightarrow \text { Walks }_{\Sigma^{\prime}},
$$

namely

$$
\mathrm{ObWalks_{ \Sigma }}=\Sigma_{0} \ni X \longmapsto f_{0}(X) \in \mathrm{ObWalks} \Sigma_{\Sigma^{\prime}}=\Sigma_{0}^{\prime}
$$

$\mathrm{Walks}_{\Sigma}(X, Y) \ni \omega=x_{1} \ldots x_{n} \longmapsto$

$$
\longmapsto \omega^{\prime}=f_{1}\left(x_{1}\right) \ldots f_{1}\left(x_{n}\right) \in \operatorname{Walks}_{\Sigma^{\prime}}\left(f_{0}(X), f_{0}(Y)\right)
$$

for any $X, Y \in \mathrm{Ob} \mathrm{Walks} \Sigma$.

1.6. Reduced walks. A walk $\omega$ on a bigraph $\Sigma$ is called reduced if it does not contain a subwalk $x x^{-1}$ for some $x \in \widehat{\Sigma}_{1}$, and reducible in opposite case. The operation

$$
\omega_{1} x x^{-1} \omega_{2} \mapsto \omega_{1} \omega_{2}
$$

will be called a reducing of the walk (by the pair $x x^{-1}$ ).

If $\omega=\omega_{1} \omega_{2}$ is a cyclic walk, then $\omega^{\prime}=\omega_{2} \omega_{1}$ is cyclic as well, and we say that $\omega$ and $\omega^{\prime}$ are cyclically equivalent. A cyclic walk $\omega$ is called cyclically reduced whenever every cyclically equivalent walk is reduced. A class $\langle\omega\rangle$ of cyclic equivalence containing the cyclic walk $\omega$ is called a cycle. Denote by $\operatorname{Cycles}_{\Sigma}$ the set of all cycles on $\Sigma$, and by RWalks $\left.\operatorname{RCycles}_{\Sigma}\right)$ the set of all reduced walks (cyclically reduced cycles) on $\Sigma$.

A bigraph morphism $f=\left(f_{0}, f_{1}\right): \Sigma \rightarrow \Sigma^{\prime}$ induces a map

$$
\operatorname{Cycles}_{\Sigma} \ni\langle\omega\rangle \stackrel{\text { Cycles }_{f}}{\longmapsto}\left\langle\operatorname{Walks}_{f}(\omega)\right\rangle \in \operatorname{Cycles}_{\Sigma^{\prime}}
$$

in a natural way.

1.7. 2-dimensional complex over a bigraph. An (abstract) 2-dimensional complex (shortly, complex) $\mathfrak{L}$ is defined by:

- a bigraph $\Sigma=\left(\Sigma_{0}, \Sigma_{1}, s, e\right.$, deg $)$ which vertices $\mathfrak{L}_{0}=\Sigma_{0}$ are considered as 0-dimensional cells, and arrows $\mathfrak{L}_{1}=\Sigma_{1}$ are considered as 1-dimensional cells;

- a set $\mathfrak{L}_{2}=\left\{\triangle_{i}, i \in I(\mathfrak{L})\right\}$ which elements are called 2-dimensional cells or 2-cells;

- a boundary map $\partial=\partial \mathfrak{L}: \mathfrak{L}_{2} \rightarrow \operatorname{RCycles}_{\Sigma}$.

We say that $A \in \mathfrak{L}_{0}\left(x \in \widehat{\mathfrak{L}}_{1}=\widehat{\Sigma}_{1}\right)$ belongs or is incident to $\triangle_{i}$ and write $A \in \triangle_{i}\left(x \in \triangle_{i}\right.$ respectively) provided $A$ (at least one of $x, x^{-1}$ respectively) is incident or belongs to some element of $\partial\left(\triangle_{i}\right)$. 
A morphism $\mathfrak{L} \rightarrow \mathfrak{L}^{\prime}$ of complexes is a triple $f=\left(f_{0}, f_{1}, f_{2}\right)$ such that $\left(f_{0}, f_{1}\right)$ is a morphism of the underlying bigraphs (denoted by the same letter $f$ ) and $f_{2}: \mathfrak{L}_{2} \rightarrow \mathfrak{L}_{2}^{\prime}$ is a map satisfying the equality

$$
\text { Cycles }_{f} \partial_{\mathfrak{L}}=\partial_{\mathfrak{L}^{\prime}} f_{2} .
$$

A morphism $f$ is called a monomorphism (epimorphism) provided all $f_{i}$ are monomorphisms (epimorphisms).

The notion of subcomplex of a complex is defined in a standard way. If $S \subset \mathfrak{L}_{0} \sqcup \mathfrak{L}_{1} \sqcup \mathfrak{L}_{2}$, then we will denote by $[S]$ the subcomplex in $\mathfrak{L}$ generated by $S$, i.e. the minimal subcomplex in $\mathfrak{L}$ containing $S$. For $S \subset \Sigma_{0}$ denote by $\Sigma_{S}$ and $\mathfrak{L}_{S}$ the corresponding restrictions of $\Sigma$ and $\mathfrak{L}$ to $S$.

Let $\mathfrak{L}$ be a complex. We will say that the complex $\mathfrak{M}$ together with a morphism $\mathrm{p}: \mathfrak{M} \rightarrow \mathfrak{L}$ form a complex over $\mathfrak{L}$. A morphism

$$
f:(\mathfrak{M}, \mathrm{p}) \rightarrow\left(\mathfrak{M}^{\prime}, \mathrm{p}^{\prime}\right)
$$

of complexes over $\mathfrak{L}$ is a morphism of complexes $f: \mathfrak{M} \rightarrow \mathfrak{M}^{\prime}$ such that $\mathrm{p}=\mathrm{p}^{\prime} f$.

1.8. Quotient complex. Let $\mathfrak{L}$ be a complex, and $\sim$ be an equivalence relation on $\mathfrak{L}_{0} \sqcup \mathfrak{L}_{1}$ such that $A \nsim a$ for any $A \in \Sigma_{0}, a \in \Sigma_{1}$, and if $a \sim b$ for some $a, b \in \Sigma_{1}$, then $\operatorname{deg}_{\Sigma}(a)=\operatorname{deg}_{\Sigma}(b), s(a) \sim s(b), e(a) \sim e(b)$. A quotient bigraph $\Sigma / \sim$ is defined by the set $\mathfrak{L}_{0} / \sim$ of vertices, the set $\mathfrak{L}_{1} / \sim$ of arrows, and correctly induced maps $e, s$ and deg.

Let $\mathrm{p}=\left(\mathrm{p}_{0}, \mathrm{p}_{1}\right): \Sigma \rightarrow \Sigma / \sim$ be the natural bigraph epimorphism. If Cycles $_{\mathfrak{p}}\left(\partial_{\mathfrak{L}}(\triangle)\right)$ is cyclically reduced for any 2-cell $\triangle \in \mathfrak{L}_{2}$, then we set

$$
(\mathfrak{L} / \sim)_{2}=\mathfrak{L}_{2} \quad \text { and } \quad \partial_{\mathfrak{L} / \sim}=\text { Cycles }_{\mathrm{p}} \partial_{\mathfrak{L}}
$$

which defines the quotient complex $\mathfrak{L} / \sim$ and the morphism

$$
\mathrm{p}=\left(\mathrm{p}_{0}, \mathrm{p}_{1}, \mathrm{p}_{2}\right): \mathfrak{L} \rightarrow \mathfrak{L} / \sim
$$

where $\mathrm{p}_{2}=1_{\mathfrak{L}_{2}}$.

1.9. Homotopy relation. A structure of 2-dimensional complex $\mathfrak{L}$ over bigraph $\Sigma$ induces the homotopy relation on Walks $\Sigma_{\Sigma}$. For any $\omega, \omega_{1}, \omega_{2} \in$ $\mathrm{Walks}_{\Sigma}$ the following transformations of walks:

1) $\omega_{1} x x^{-1} \omega_{2} \rightsquigarrow \omega_{1} \omega_{2}$ or $\omega_{1} \omega_{2} \rightsquigarrow \omega_{1} x x^{-1} \omega_{2}$ for any $x \in \widehat{\Sigma}_{1}$;

2) $\omega_{1} \omega_{2} \rightsquigarrow \omega_{1} \omega \omega_{2}$ or $\omega_{1} \omega \omega_{2} \rightsquigarrow \omega_{1} \omega_{2}$ such that $\langle\omega\rangle=\partial(\triangle), \triangle \in \mathfrak{L}_{2}$;

are called elementary homotopies.

Two walks $\omega, \omega^{\prime}$ on $\Sigma$ are said to be homotopic provided there exists a sequence $E=\left(E_{1}, \ldots, E_{N}\right)$ of elementary homotopies such that

$$
\omega=\omega_{0} \stackrel{E_{1}}{\rightsquigarrow} \omega_{1} \stackrel{E_{2}}{\rightsquigarrow} \ldots \stackrel{E_{N}}{\rightsquigarrow} \omega_{N}=\omega^{\prime}, N \geqslant 0 .
$$


In this case, we write $\omega \stackrel{E}{\rightsquigarrow} \omega^{\prime}$ or $\omega \sim \omega^{\prime}$ and say that $E$ is a homotopy between $\omega$ and $\omega^{\prime}$.

We indicate the simple properties of homotopies:

1) the relation $\sim$ is an equivalence;

2) if $\omega \sim \omega^{\prime}$, then $s(\omega)=s\left(\omega^{\prime}\right), e(\omega)=e\left(\omega^{\prime}\right)$;

3) if $\omega_{1} \sim \omega_{1}^{\prime}, \omega_{2} \sim \omega_{2}^{\prime}$, then $\omega_{1} \omega_{2} \sim \omega_{1}^{\prime} \omega_{2}^{\prime}$ provided one of the compositions is defined.

For a walk $\omega$ we denote by $[\omega]$ the homotopic class of $\omega$. Once the composition $\omega \omega^{\prime}$ of walks $\omega, \omega^{\prime}$ is specified, the composition of their classes is correctly defined by the equality $[\omega] \cdot\left[\omega^{\prime}\right]=\left[\omega \omega^{\prime}\right]$. We will denote by Hot $_{\mathfrak{L}}$ the quotient category of homotopic classes of walks on $\Sigma$,

$$
\operatorname{Hot}_{\mathfrak{L}}(X, Y)=\operatorname{Walks}_{\Sigma}(X, Y) / \sim .
$$

Note that $\operatorname{Hot}_{\mathfrak{L}}$ is a groupoid.

1.10. Fundamental group of a complex. A cyclic walk on $\mathfrak{L}$ is called contractible if it is homotopic to a trivial walk. For any $B \in \mathfrak{L}_{0}$, we define the fundamental group $G(\mathfrak{L}, B)$ of $\mathfrak{L}$ with the base vertex $B$ as $\operatorname{Hot}_{\mathfrak{L}}(B, B)$. If $\mathfrak{L}$ is connected, then the fundamental groups with different base vertices are isomorphic $([22])$, which allows to define the fundamental group $G(\mathfrak{L})$ of the connected complex $\mathfrak{L}$.

1.11. Covering of a complex. An epimorphism $\mathrm{p}: \widetilde{\mathfrak{L}} \rightarrow \mathfrak{L}$ of complexes is called a covering morphism (or covering of $\mathfrak{L}$ ) with the base $\mathfrak{L}$, if:

- for every $\omega \in \operatorname{Walks}_{\mathfrak{L}}(X, Y)$ and $\tilde{X} \in \mathrm{p}_{0}^{-1}(X)\left(\tilde{Y} \in \mathrm{p}_{0}^{-1}(Y)\right)$, there exist an unique $\tilde{Y} \in \widetilde{\mathfrak{L}}_{0}\left(\tilde{X} \in \widetilde{\mathfrak{L}}_{0}\right)$ and a unique walk $\widetilde{\omega}: \widetilde{X} \rightarrow \widetilde{Y}$ such that $\mathrm{Walks}_{\mathrm{p}}(\widetilde{\omega})=\omega$ (the property of the uniqueness of lifting of walks, [22]);

- for every $\triangle \in \mathfrak{L}_{2}, A \in \mathfrak{L}_{0}$ such that $A \in \partial(\triangle)$ and $\widetilde{A} \in \mathrm{p}_{0}^{-1}(A)$ there exists unique $\widetilde{\triangle} \in \widetilde{\mathfrak{L}}_{2}$ such that $\widetilde{A} \in \partial(\widetilde{\triangle})$ and $\mathrm{p}_{2}(\widetilde{\triangle})=\triangle$ (the property of homotopy lifting uniqueness, [22]).

Coverings with a fixed base $\mathfrak{L}$ form the subcategory in the category of complexes over $\mathfrak{L}$. An object $p: \widetilde{\mathfrak{L}} \rightarrow \mathfrak{L}$ in this category is called a universal covering of $\mathfrak{L}$ if every morphism $f: \mathrm{p}^{\prime} \rightarrow \mathrm{p}$ is an isomorphism.

1.12. Construction of the universal covering. We will now construct the universal covering $\widetilde{\mathfrak{L}}$ of a complex $\mathfrak{L}$ similarly to $[1,11]$.

1) Fix $B \in \mathfrak{L}_{0}$ and define the set $\widetilde{\mathfrak{L}}_{0}$ by $\sqcup_{X \in \mathfrak{L}_{0}} \operatorname{Hot}_{\mathfrak{L}}(B, X)$.

2) The arrows in $\widetilde{\mathfrak{L}}$ are the pairs $([\omega], x)$ of $[\omega] \in \widetilde{\mathfrak{L}}_{0}, x \in \mathfrak{L}_{1}$ such that

$$
\begin{array}{ll}
s(x)=e(\omega), & \operatorname{deg}_{\mathfrak{L}}(([\omega], x))=\operatorname{deg}_{\mathfrak{L}}(x), \\
s(([\omega], x))=[\omega], & e(([\omega], x))=[x \omega] .
\end{array}
$$


3) The covering morphism $\mathrm{p}: \tilde{\Sigma} \rightarrow \Sigma$ on the underlying bigraphs is defined by equalities

$$
\mathrm{p}_{0}([\omega])=e(\omega), \quad \mathrm{p}_{1}(([\omega], x))=x .
$$

Obviously, the constructed morphism $\mathrm{p}$ has a property of the uniqueness of lifting of walks.

4) If $\triangle \in \mathfrak{L}_{2}$ and $\langle\omega\rangle=\partial_{\mathfrak{L}}(\triangle)$, then every walk $\tilde{\omega} \in \operatorname{Walks}_{\mathrm{p}}^{-1}(\omega)$ is also cyclic since $\omega$ is contractible on $\mathfrak{L}$. Let

$$
\Theta=\underset{\triangle \in \mathfrak{L}_{2}}{\sqcup} \operatorname{Cycles}_{\mathrm{p}}^{-1}\left(\partial_{\mathfrak{L}}(\triangle)\right) .
$$

Then we define $\widetilde{\mathfrak{L}}_{2}$ by the set $\left\{\tilde{\triangle}_{\theta}\right\}_{\theta \in \Theta}$, the boundary map in $\tilde{\mathfrak{L}}$ by the equality $\partial_{\mathfrak{L}}\left(\tilde{\triangle}_{\theta}\right)=\theta$, and $\mathrm{p}_{2}\left(\widetilde{\triangle}_{\theta}\right)=\triangle$ provided $\operatorname{Cycles}_{\mathrm{p}}(\theta)=\partial_{\mathfrak{L}}(\triangle)$.

The fundamental group $G=G(\mathfrak{L}, B)$ acts on $\widetilde{\mathfrak{L}}$ as follows:

$$
\text { if }\left[\omega_{B}\right] \in G(\mathfrak{L}, B),[\omega] \in \widetilde{\mathfrak{L}}_{0} \text {, then }[\omega]\left[\omega_{B}\right]=\left[\omega \omega_{B}\right] \in \widetilde{\mathfrak{L}}_{0} .
$$

The structure maps of $\tilde{\mathfrak{L}}$ commutes with the action of $G$, hence a quotient complex

$$
\tilde{\mathfrak{L}} / G=\left(G \widetilde{\mathfrak{L}}_{0}, G \widetilde{\mathfrak{L}}_{1}, G \widetilde{\mathfrak{L}}_{2}\right)
$$

is defined, and the morphism $\mathrm{p}$ induces an isomorphism of the complexes $\tilde{\mathfrak{L}} / G$ and $\mathfrak{L}$.

\section{Bimodule problems}

2.1. Main definitions. Let $\mathbf{k}$ be an algebraically closed field. Unless otherwise stated, all the categories we consider are the categories over $\mathbf{k}$, all morphism spaces are finite dimensional, and all functors are $\mathbf{k}$-linear.

A category $\mathrm{K}$ is called local provided for every $X \in \mathrm{ObK}$ the endomorphism algebra $\mathrm{K}(X, X)$ is local, and regular, if, in addition, every invertible morphism is automorphism.

A category $\mathrm{K}$ is called fully additive or Krull-Schmidt category if $\mathrm{K}$ is a category with finite direct sums and every idempotent from $\mathrm{K}$ splits, i.e. it has kernel and cokernel. We call an object $X$ of additive category indecomposable whenever $X \not X_{1} \oplus X_{2}$ for any non-zero objects $X_{1}, X_{2}$.

For a local category $\mathrm{K}$ and for every $X \in \mathrm{Ob} \mathrm{K}$, there exists the decomposition $\mathrm{K}(X, X)=\mathbf{k} \mathbb{1}_{X} \oplus \operatorname{Rad} X$ where $\operatorname{Rad} X$ is the Jacobson radical of the algebra $\mathrm{K}(X, X)$. If $\mathrm{K}$ is regular, then we denote by RadK the radical of $\mathrm{K}$, i.e. an ideal in $\mathrm{K}$ such that

$$
\operatorname{RadK}(X, Y)=\mathrm{K}(X, Y) \text { for } X \neq Y,
$$

and

$$
\operatorname{RadK}(X, X)=\operatorname{Rad} X, X, Y \in \mathrm{ObK}
$$


Let $\mathrm{V}$ be a $\mathrm{K}$-bimodule ([1]). A category $\mathrm{K}$ (a bimodule $\mathrm{V}$ ) is called locally finite dimensional, if the spaces

$$
\begin{gathered}
\underset{Y \in \mathrm{ObK}}{\oplus} \mathrm{K}(X, Y) \text { and } \underset{Y \in \mathrm{Ob} \mathrm{K}}{\oplus} \mathrm{K}(Y, X) \\
\text { (resp. } \underset{Y \in \mathrm{ObK}}{\oplus} \mathrm{V}(X, Y) \text { and } \underset{Y \in \mathrm{Ob} \mathrm{K}}{\oplus} \mathrm{V}(Y, X))
\end{gathered}
$$

are finite dimensional for any $X \in \mathrm{ObK}$, and finite dimensional provided $|\mathrm{ObK}|<\infty$, and the spaces

$$
\underset{X, Y \in \mathrm{Ob} \mathrm{K}}{\oplus} \mathrm{K}(X, Y) \quad(\text { resp. } \underset{X, Y \in \mathrm{Ob} \mathrm{K}}{\oplus} \mathrm{V}(X, Y))
$$

are finite dimensional.

Definition 2.2. A pair $\mathcal{A}=(\mathrm{K}, \mathrm{V})$ consisting of a category $\mathrm{K}$ and a $\mathrm{K}$ bimodule $\mathrm{V}$ is called a bimodule problem over $\mathrm{K}$ or shortly bimodule problem. Given two bimodule problems $\mathcal{A}=(\mathrm{K}, \mathrm{V})$ and $\mathcal{A}^{\prime}=\left(\mathrm{K}^{\prime}, \mathrm{V}^{\prime}\right)$, a morphism of bimodule problems $\theta: \mathcal{A} \rightarrow \mathcal{A}^{\prime}$ is a pair $\theta=\left(\theta_{0}, \theta_{1}\right)$ where $\theta_{0}: \mathrm{K} \rightarrow \mathrm{K}^{\prime}$ is a $\mathbf{k}$-functor, and $\theta_{1}: \mathrm{V} \rightarrow \mathrm{V}^{\prime}$ is a $\mathrm{K}$-bimodule morphism with the $\mathrm{K}$-bimodule structure on $\mathrm{V}^{\prime}$ induced by $\theta_{0}$ ([1]).

A bimodule problem $\mathcal{A}$ is called (locally) finite dimensional provided both $\mathrm{K}$ and $\mathrm{V}$ are (locally) finite dimensional.

A locally finite dimensional bimodule problem $\mathcal{A}$ over a regular category $\mathrm{K}$ will be called normal. All the bimodule problems we will consider are assumed to be normal.

Given some $S \subset \mathrm{ObK}$ denote by $\mathrm{K}_{S}$ the full subcategory of $\mathrm{K}$ with Ob $\mathrm{K}_{S}=S$, and by $\mathrm{V}_{S}$ the subbimodule $\mathrm{V}_{S}=\mathrm{K}_{S} \mathrm{VK}_{S}$. A bimodule problem $\mathcal{A}_{S}=\left(\mathrm{K}_{S}, \mathrm{~V}_{S}\right)$ is called a restriction of $\mathcal{A}$ to $S$.

For a category $\mathrm{K}$, we denote by add $\mathrm{K}$ an additive hull of $\mathrm{K}$, i.e. a minimal fully additive category which contains $\mathrm{K}$. For a K-bimodule $\mathrm{V}$, we denote by add $\mathrm{V}$ and by add $\mathcal{A}$ the corresponding add K-bimodule and bimodule problem (add $\mathrm{K}$, add $\mathrm{V}$ ) respectively.

Let $\mathrm{V}$ be a $\mathrm{K}$-bimodule. We say that $x \in \operatorname{RadK}(X, Y)$ annihilates the bimodule $\mathrm{V}$ if $x a=0, b x=0$ for any $a \in \mathrm{V}(Z, X), b \in \mathrm{V}(Y, Z), Z \in \mathrm{ObK}$. The ideal of $\mathrm{K}$ consisting of all elements annihilating the bimodule $\mathrm{V}$ is called the annihilator of $\mathrm{V}$ and is denoted by $\mathrm{Ann} n_{\mathrm{K}} \mathrm{V}$. A bimodule problem $\mathcal{A}=(\mathrm{K}, \mathrm{V})$ is called faithful if $\mathrm{Ann} \mathrm{K} \mathrm{V}=0$.

Let $\mathcal{A}=(\mathrm{K}, \mathrm{V})$ be a bimodule problem, and $\mathrm{V}^{\prime} \subset \mathrm{V}$ be a subbimodule of $\mathrm{V}$ such that $\mathrm{V}^{\prime} \neq 0, \mathrm{~V}^{\prime} \neq \mathrm{V}$. Denote by $>$ the minimal relation of (strict) partial order on the set of bimodule problems such that $\mathcal{A}>\mathcal{A}^{\prime}$ and $\mathcal{A}>\mathcal{A}^{\prime \prime}$, where $\mathcal{A}^{\prime} \simeq\left(\mathrm{K}, \mathrm{V}^{\prime}\right), \mathcal{A}^{\prime \prime} \simeq\left(\mathrm{K}, \mathrm{V} / \mathrm{V}^{\prime}\right)$, and $\mathcal{A}>\mathcal{A}_{S}$ for any proper subset $S \subset \mathrm{ObK}$. Similarly we denote by $\sim$ the minimal equivalence 
such that $\mathcal{A} \sim \mathcal{A}_{\mathcal{I}}=(\mathrm{K} / \mathcal{I}, \mathrm{V})$ for every ideal $\mathcal{I} \subset \mathrm{Ann}_{\mathrm{K}} \mathrm{V}$, and if $\mathcal{A} \simeq \mathcal{B}$, then $\mathcal{A} \sim \mathcal{B}$. The transitive closure of $>$ and $\sim$ defines a preorder on the set of bimodule problems, which defines the strict order, denoted by $>$ again. The relations $>$ and $\sim$ are obviously defined on the set of isoclasses of bimodule problems. If $\mathcal{A}>\mathcal{B}$ for bimodule problems $\mathcal{A}$ and $\mathcal{B}$, then we say that $\mathcal{B}$ is a subproblem of $\mathcal{A}$.

2.3. Basis of bimodule problem. A bigraph $\Sigma\left(=\Sigma_{\mathcal{A}}\right)$ is called a basis of a normal bimodule problem $\mathcal{A}=(\mathrm{K}, \mathrm{V})$ if $\Sigma_{0}=\mathrm{ObK}, \Sigma_{1}^{0}(X, Y)$ is a basis of $\mathrm{V}(X, Y)$, and $\Sigma_{1}^{1}(X, Y)$ is a basis of $\operatorname{RadK}(X, Y), X, Y \in \mathrm{ObK}$. A bimodule problem is called connected, if its bigraph is connected.

2.4. Tits quadratic form of bimodule problem. A quadratic form $q=$ $q_{\Sigma}$ of a bigraph $\Sigma$ is defined on $x=\left(x_{A}\right)_{A \in \Sigma_{0}} \in \mathbb{Z}^{\Sigma_{0}}$ by equality

$$
q(x)=\sum_{(A, B) \in \Sigma_{0} \times \Sigma_{0}}\left(\delta_{A B}+\left|\Sigma_{1}^{1}(A, B)\right|-\left|\Sigma_{1}^{0}(A, B)\right|\right) x_{A} x_{B}
$$

where $\delta$ is the Kronecker delta. By definition, the Tits quadratic form $q_{\mathcal{A}}$ of a bimodule problem $\mathcal{A}$ is a quadratic form $q_{\Sigma_{\mathcal{A}}}$ of a basic bigraph $\Sigma_{\mathcal{A}}$.

2.5. Representation category. For a bimodule problem $\mathcal{A}=(\mathrm{K}, \mathrm{V}), a$ representation $M$ of $\mathcal{A}$ is a pair

$$
M=\left(M_{\mathrm{K}}, M_{\mathrm{V}}\right),
$$

where $M_{\mathrm{K}} \in \mathrm{Ob}$ add $\mathrm{V}=\mathrm{Ob}$ add $\mathrm{K}$ and $M_{\mathrm{V}} \in \operatorname{add} \mathrm{V}\left(M_{\mathrm{K}}, M_{\mathrm{K}}\right)$. If $M, N$ are two representations of $\mathcal{A}$, then a morphism $f$ from $M$ to $N$ is a morphism $f \in \operatorname{add} \mathrm{K}\left(M_{\mathrm{K}}, N_{\mathrm{K}}\right)$ such that $N_{\mathrm{V}} f-f M_{\mathrm{V}}=0$. The unit morphisms and composition of morphisms in the representation category $\operatorname{rep} \mathcal{A}$ and in the category add $\mathrm{K}$ coincide. All indecomposable representations form the subcategory in $\operatorname{rep} \mathcal{A}$ which we denote by ind $\mathcal{A}$.

With a locally finite dimensional bimodule problem $\mathcal{A}=(\mathrm{K}, \mathrm{V})$ we associate the $\mathbb{Z}$-lattice

$$
\operatorname{dim}_{\mathcal{A}}=\underset{\mathrm{ObK}}{\oplus} \mathbb{Z}
$$

of elements $x=\left(x_{A}\right)_{A \in \mathrm{ObK}}$ with finite support

$$
\operatorname{supp} x=\left\{A \in \mathrm{ObK} \mid x_{A} \neq 0\right\} .
$$

The lattice $\operatorname{dim}_{\mathcal{A}}$ has the standard basis $\left\{e_{A}, A \in \mathrm{ObK}\right\}$ such that $\left(e_{A}\right)_{A}=1$, and $\left(e_{A}\right)_{B}=0$ for $B \in \mathrm{Ob} \mathrm{K} \backslash\{A\}$. Besides, $\operatorname{dim}_{\mathcal{A}}$ is endowed with the partial product order: for a vector $x \in \operatorname{dim}_{\mathcal{A}}$, we write $x \geqslant 0$ if and only if $x_{A} \geqslant 0$ for all $A \in \mathrm{ObK}$. For a representation $M \in \operatorname{rep} \mathcal{A}$ such that

$$
M_{\mathrm{K}} \simeq \underset{A \in \mathrm{ObK}}{\oplus} A^{x_{A}}
$$


where almost all $x_{A}=0$, a dimension vector of $M$ is defined by equality

$$
\operatorname{dim} M=\operatorname{dim}_{\mathcal{A}} M=\left(x_{A}\right)_{A \in \mathrm{ObK}} \in \operatorname{dim}_{\mathcal{A}} .
$$

By definition, a support $\operatorname{supp} M$ of the representation $M$ is $\operatorname{supp}_{\operatorname{dim}_{\mathcal{A}}} M$ and is always finite.

The category rep $\mathcal{A}$ is Krull-Schmidt category. Bimodule problem $\mathcal{A}$ is called of finite representation type provided $\operatorname{rep} \mathcal{A}$ has finitely many isoclasses of indecomposable objects, and of infinite representation type in opposite case. $\mathcal{A}$ is called locally representation-finite provided for any object $A \in \mathrm{ObK}$, there are finitely many isoclasses of indecomposable representations $M$ such that $A \in \operatorname{supp} M$.

A representation $M \in \operatorname{rep} \mathcal{A}$ is called sincere provided $(\operatorname{dim} M)_{A} \neq 0$ for any $A \in \mathrm{ObK}$. A bimodule problem $\mathcal{A}$ is called sincere if there exists a sincere indecomposable representation $M \in \operatorname{rep} \mathcal{A}$.

A representation $M \in \operatorname{ind} \mathcal{A}$ is called schurian provided it has only scalar endomorphisms. A bimodule problem $\mathcal{A}$ is called schurian provided every $M \in \operatorname{ind} \mathcal{A}$ is schurian $([14,17])$.

Lemma 2.6 ([13,17]). Let $\mathcal{A}$ be a finite dimensional schurian bimodule problem. Then $\mathcal{A}$ is representation finite, its Tits form $q_{\mathcal{A}}$ is unit integral and $W P$, the map $\operatorname{dim}_{\mathcal{A}}: \operatorname{ind} \mathcal{A} / \simeq \rightarrow \Re_{q_{\mathcal{A}}}^{+}$is a bijection, where ind $\mathcal{A} / \simeq$ denotes the set of all isoclasses of indecomposable representations.

2.7. Covering of bimodule problem. A bimodule problem $\tilde{\mathcal{A}}=(\tilde{\mathrm{K}}, \tilde{\mathrm{V}})$ together with a bimodule problem morphism $\mathrm{p}=\left(\mathrm{p}_{0}, \mathrm{p}_{1}\right): \tilde{\mathcal{A}} \rightarrow \mathcal{A}$ is called a covering of a bimodule problem $\mathcal{A}=(\mathrm{K}, \mathrm{V})$ provided $\mathrm{p}_{0}$ is a surjection on the objects and $\mathrm{p}_{0}, \mathrm{p}_{1}$ induce the following isomorphisms for any $\tilde{A}, \tilde{B} \in$ $\operatorname{Ob} \tilde{K}([11])$ :

$$
\begin{aligned}
& \underset{\tilde{X} \in \mathrm{p}_{0}^{-1}\left(\mathrm{p}_{0}(\tilde{B})\right)}{\oplus} \tilde{\mathrm{K}}(\tilde{A}, \tilde{X}) \simeq \mathrm{K}\left(\mathrm{p}_{0}(\tilde{A}), \mathrm{p}_{0}(\tilde{B})\right), \\
& \underset{\tilde{Y} \in \mathrm{p}_{0}^{-1}\left(\mathrm{p}_{0}(\tilde{A})\right)}{\oplus} \tilde{\mathrm{K}}(\tilde{Y}, \tilde{B}) \simeq \mathrm{K}\left(\mathrm{p}_{0}(\tilde{A}), \mathrm{p}_{0}(\tilde{B})\right), \\
& \underset{\tilde{X} \in \mathrm{p}_{0}^{-1}\left(\mathrm{p}_{0}(\tilde{B})\right)}{\oplus} \tilde{\mathrm{V}}(\tilde{A}, \tilde{X}) \simeq \mathrm{V}\left(\mathrm{p}_{0}(\tilde{A}), \mathrm{p}_{0}(\tilde{B})\right), \\
& \underset{\tilde{Y} \in \mathrm{p}_{0}^{-1}\left(\mathrm{p}_{0}(\tilde{A})\right)}{\bigoplus} \tilde{\mathrm{V}}(\tilde{Y}, \tilde{B}) \simeq \mathrm{V}\left(\mathrm{p}_{0}(\tilde{A}), \mathrm{p}_{0}(\tilde{B})\right) .
\end{aligned}
$$

In this case the bimodule problem $\mathcal{A}$ is called a base of the covering and $\mathrm{p}$ is called a covering morphism.

The coverings with a fixed base form a category over $\mathcal{A}$ in a standard way: a morphism of the coverings $\mathrm{p}: \tilde{\mathcal{A}} \rightarrow \mathcal{A}$ and $\mathrm{p}^{\prime}: \tilde{\mathcal{A}}^{\prime} \rightarrow \mathcal{A}$ is a morphism of bimodule problems $\rho: \tilde{\mathcal{A}} \rightarrow \tilde{\mathcal{A}}^{\prime}$ such that $\mathrm{p}=\mathrm{p}^{\prime} \rho$. 
The functor

$$
\mathrm{p}_{*}=\operatorname{rep} \mathrm{p}: \operatorname{rep} \tilde{\mathcal{A}} \rightarrow \operatorname{rep} \mathcal{A}
$$

between the representation categories induced by the morphism $\mathrm{p}: \tilde{\mathcal{A}} \rightarrow \mathcal{A}$ of bimodule problems is called the push-down functor, ([12]). It allows to compare the representation types of bimodule problems $\tilde{\mathcal{A}}$ and $\mathcal{A}$.

2.8. Galois covering. Let $\tilde{\mathcal{A}}=(\tilde{\mathrm{K}}, \tilde{\mathrm{V}})$ be a locally finite dimensional bimodule problem, let $G$ be a group acting freely on $\tilde{\mathrm{K}}$ and $\tilde{\mathrm{V}}$ that means:

- there is given a group monomorphism

$$
T: G \rightarrow \operatorname{Aut}_{\mathbf{k}}(\tilde{\mathrm{K}}, \tilde{\mathrm{K}})
$$

which defines a free action

$$
T_{\tilde{\mathrm{K}}}: G \times \tilde{\mathrm{K}} \rightarrow \tilde{\mathrm{K}}
$$

of $G$ on $\tilde{\mathrm{K}}$ such that

$$
G \times \mathrm{Ob} \tilde{\mathrm{K}} \ni(g, A) \stackrel{T_{\tilde{\mathrm{K}}}}{\longmapsto} g A=T(g)(A) \in \mathrm{Ob} \tilde{\mathrm{K}} ;
$$

- an action

$$
T_{\tilde{\mathrm{V}}}: G \times \tilde{\mathrm{V}} \rightarrow \tilde{\mathrm{V}}
$$

of $G$ on $\tilde{V}$ is a family of $\mathbf{k}$-isomorphisms

$$
T_{\tilde{\mathrm{V}}}(g)(A, B): \tilde{\mathrm{V}}(A, B) \rightarrow \tilde{\mathrm{V}}(g A, g B)
$$

such that

$$
T_{\tilde{\mathbf{V}}}(g)\left(f_{1} v f_{2}\right)=T_{\tilde{\mathbf{K}}}(g)\left(f_{1}\right) T_{\tilde{\mathbf{V}}}(g)(v) T_{\tilde{\mathbf{K}}}(g)\left(f_{2}\right)
$$

for any $f_{1}, f_{2} \in \tilde{\mathrm{K}}, v \in \tilde{\mathrm{V}}$ and $g \in G$ once the composition $f_{1} v f_{2}$ is specified. For a locally finite dimensional bimodule problem $\tilde{\mathcal{A}}=(\tilde{\mathrm{K}}, \tilde{\mathrm{V}})$ and a group $G$ acting freely on $\tilde{\mathrm{K}}$ and $\tilde{\mathrm{V}}$, we construct the bimodule problem $\mathcal{A}=(\mathrm{K}, \mathrm{V})$ and a covering morphism $\mathrm{p}=\left(\mathrm{p}_{0}, \mathrm{p}_{1}\right): \tilde{\mathcal{A}} \rightarrow \mathcal{A}$ in a following way. Let the set $\mathrm{ObK}$ be the set $(\mathrm{Ob} \tilde{\mathrm{K}}) / G$ of orbits, and the functor $\mathrm{p}_{0}$ be the natural projection $\mathrm{Ob} \tilde{\mathrm{K}} \rightarrow \mathrm{ObK}$ on objects. For objects $\tilde{A}, \tilde{B} \in \mathrm{Ob} \tilde{\mathrm{K}}$, identify an element $\varphi \in \tilde{\mathrm{K}}(\tilde{A}, \tilde{B})(a \in \tilde{\mathrm{V}}(\tilde{A}, \tilde{B})$ respectively) with the corresponding element of the sum

$$
\begin{gathered}
\oplus_{\tilde{X} \in \mathrm{p}_{0}^{-1}\left(\mathrm{p}_{0}(\tilde{A})\right), \tilde{Y} \in \mathrm{p}_{0}^{-1}\left(\mathrm{p}_{0}(\tilde{B})\right)} \tilde{\mathrm{K}}(\tilde{X}, \tilde{Y}) \\
\left(\operatorname{resp} . \oplus_{\tilde{X} \in \mathrm{p}_{0}^{-1}\left(\mathrm{p}_{0}(\tilde{A})\right), \tilde{Y} \in \mathrm{p}_{0}^{-1}\left(\mathrm{p}_{0}(\tilde{B})\right)} \tilde{\mathrm{V}}(\tilde{X}, \tilde{Y})\right) .
\end{gathered}
$$

For $A, B \in \mathrm{Ob} \mathrm{K}$, we define

$$
\mathrm{K}(A, B)=\left(\underset{\tilde{X} \in \mathrm{p}_{0}^{-1}(A), \tilde{Y} \in \mathrm{p}_{0}^{-1}(B)}{\oplus} \tilde{\mathrm{K}}(\tilde{X}, \tilde{Y})\right) / \tilde{\mathrm{K}}_{G},
$$




$$
\mathrm{V}(A, B)=\left(\underset{\tilde{X} \in \mathrm{p}_{0}^{-1}(A), \tilde{Y} \in \mathrm{p}_{0}^{-1}(B)}{\bigoplus_{\mathrm{V}}}(\tilde{X}, \tilde{Y})\right) / \tilde{\mathrm{V}}_{G},
$$

where $\tilde{\mathrm{K}}_{G}\left(\tilde{\mathrm{V}}_{G}\right.$ respectively) is the subspace generated by $\varphi-g \varphi(a-g a)$ provided $\varphi(a)$ runs $\tilde{\mathrm{K}}(\tilde{X}, \tilde{Y})(\tilde{\mathrm{V}}(\tilde{X}, \tilde{Y}))$ for all $\tilde{X}, \tilde{Y} \in \mathrm{Ob} \tilde{\mathrm{K}}$ such that $\mathrm{p}_{0}(\tilde{X})=A, \mathrm{p}_{0}(\tilde{Y})=B$, and $g$ runs $G$. Denote the class of $\varphi(a)$ by $G \varphi \in \mathrm{K}(A, B)(G a \in \mathrm{V}(A, B))$.

For $\varphi \in \tilde{\mathrm{K}}(\tilde{X}, \tilde{Y}), \psi \in \tilde{\mathrm{K}}(g \tilde{Y}, \tilde{Z})$, the composition of $G \varphi$ and $G \psi$ is defined by $G(b(g a))$, and the sum of $G a$ and $G c$ for $c \in \tilde{\mathrm{K}}(g \tilde{X}, g \tilde{Y})$ is $G(g a+c)$. Now we can define the functor $\mathrm{p}_{0}$ on morphisms by the map

$$
\tilde{\mathrm{K}}(\tilde{A}, \tilde{B}) \ni \varphi \longmapsto G \varphi \in \mathrm{K}\left(\mathrm{p}_{0}(\tilde{A}), \mathrm{p}_{0}(\tilde{B})\right) .
$$

The K-bimodule structure on $\mathrm{V}$ and the map $\mathrm{p}_{1}$ are defined similarly.

The bimodule problem $\mathcal{A}$ is called a G-quotient of $\tilde{\mathcal{A}}$ and is denoted by $\tilde{\mathcal{A}} / G$. The constructed morphism $\mathrm{p}_{G}=\mathrm{p}: \tilde{\mathcal{A}} \rightarrow \mathcal{A}$ of bimodule problems mapping an object $\tilde{X}$ to $G \tilde{X}$ and an arrow $a: \tilde{X} \rightarrow \tilde{Y}$ to $G a: G \tilde{X} \rightarrow G \tilde{Y}$ is correctly defined, is a covering morphism and is called a quotient morphism of the bimodule problems.

A covering isomorphic to the defined above covering $\mathrm{p}_{G}: \tilde{\mathcal{A}} \rightarrow \tilde{\mathcal{A}} / G$ is called a Galois covering with the fundamental group $G$.

Theorem 2.9 ([12]). Let $\mathrm{p}_{G}: \tilde{\mathcal{A}} \rightarrow \mathcal{A}$ be a Galois covering of a bimodule problem $\mathcal{A}$ with a fundamental group $G$, and let the covering $\tilde{\mathcal{A}}$ be locally representation-finite. Then the push-down functor

$$
\operatorname{rep~p} \mathrm{p}_{G}: \operatorname{rep} \tilde{\mathcal{A}} \rightarrow \operatorname{rep} \mathcal{A}
$$

is a Galois covering of $\operatorname{rep} \mathcal{A}$ with the fundamental group $G$, and $\mathcal{A}$ is locally representation-finite. If $\mathcal{A}$ is finite dimensional, then $\mathcal{A}$ is of finite representation type.

\section{ONE-SIDED BIMODULE PROBLEMS}

3.1. Triangled basis. Let $\mathcal{A}=(\mathrm{K}, \mathrm{V})$ be a normal bimodule problem, $\Sigma$ be a basis of $\mathcal{A}$. Assume that the radical $\mathrm{R}=\mathrm{RadK}$ is nilpotent. The integer $N$ is called the nilpotence degree of $\mathcal{A}$ if $\mathrm{R}^{N}=0$, but $\mathrm{R}^{N-1} \neq 0$.

Denote by $\mathrm{V}_{i}=\mathrm{R}^{i-1} \mathrm{~V}, i=1, \ldots, N$. Then we have two filtrations

$$
\mathrm{R} \supset \mathrm{R}^{2} \supset \ldots \supset \mathrm{R}^{N-1} \supset 0, \mathrm{~V}_{1} \supset \mathrm{V}_{2} \supset \ldots \supset \mathrm{V}_{N} \supset 0
$$

Notice that all inclusions are strict here, and for a faithful $\mathcal{A}, V_{i} \neq 0$ for all $i=1, \ldots, N$.

The map $h: \mathrm{R} \cup \mathrm{V} \rightarrow \mathbb{N}$ defined by

$$
h(x)=\max \left\{i \in \mathbb{N} \mid x \in \mathrm{R}^{i} \cup \mathrm{V}_{i}\right\}
$$


is called the height of an element. Then the set

$$
\left\{\Sigma_{1}^{k^{(i)}}=\Sigma_{1}^{k} \cap h^{-1}(i), i=1, \ldots, N\right\}
$$

is a partition of $\Sigma_{1}^{k}, k=0,1$.

A basis $\Sigma$ of a bimodule problem $\mathcal{A}$ will be called triangled (with respect to the filtration), whenever $\cup_{l=i}^{N-1} \Sigma_{1}^{1^{(l)}}$ is a basis of $\mathrm{R}^{i}$ and $\bigcup_{l=i}^{N} \Sigma_{1}^{0^{(l)}}$ is a basis of $\mathrm{V}_{i}, i=1, \ldots, N$. Every normal finite dimensional bimodule problem $\mathcal{A}$ with nilpotent radical has a triangled basis ([10]).

3.2. One-sided bimodule problem. A normal bimodule problem $\mathcal{A}$ with a nilpotent radical $\mathrm{R}=\mathrm{RadK}$ will be called one-sided or admitted if the set $\mathrm{ObK}$ can be decomposed to a disjoint union

$$
\mathrm{ObK}=\mathrm{ObK}^{+} \cup \mathrm{ObK}^{-}
$$

such that inequality $\mathrm{V}(X, Y) \neq 0$ implies $X \in \mathrm{ObK}^{-}, Y \in \mathrm{ObK}^{+}$, and $\mathrm{R}(X, Y) \neq 0$ implies $X, Y \in \mathrm{Ob}^{+}$. The property of a bimodule problem $\mathcal{A}$ to be admitted depends only on the bigraph $\Sigma_{\mathcal{A}}$, and we denote by $\Sigma_{0}^{+}=\mathrm{ObK}^{+}$and $\Sigma_{0}^{-}=\mathrm{ObK}^{-}$.

Let $\mathcal{A}=(\mathrm{K}, \mathrm{V})$ be an admitted bimodule problem with nilpotent radical $\mathrm{R}=$ RadK and a triangled basis $\Sigma$.

For $a, b \in \mathrm{V}$, we say that $a<_{\mathrm{R}} b$ if $b \in \mathrm{R} a$. Two elements $a, b \in \mathrm{V}$ are called comparable is either $a<_{\mathrm{R}} b$ or $b<_{\mathrm{R}} a$. For $A \in \Sigma_{0}^{+}$let

$$
\operatorname{ord} A=\sum_{E \in \Sigma_{0}^{-}} \operatorname{dim}_{\mathbf{k}} \mathrm{V}(E, A)=\sum_{E \in \Sigma_{0}^{-}}\left|\Sigma_{1}(E, A)\right| .
$$

3.3. The considered class $C$. Define the class $C$ of one-sided bimodule problems $\mathcal{A}=(\mathrm{K}, \mathrm{V})$ with nilpotent radical $\mathrm{R}$ and a triangled basis $\Sigma$ such that for any $E \in \Sigma_{0}^{-}, A, B \in \Sigma_{0}^{+}, A \neq B$ :

1) $\operatorname{ord} A \leqslant 3$

2) any $a_{1}, a_{2} \in \Sigma_{1}^{0}(E, A)$ are comparable;

3) if $\operatorname{ord} A=\operatorname{ord} B=3$, then any $a \in \Sigma_{1}^{0}(E, A), b \in \Sigma_{1}^{0}(E, B)$ are comparable;

4) if $\varphi \in \mathrm{R}(A, B)$, then $\sum_{E \in \Sigma_{0}^{-}} \operatorname{dim}_{\mathrm{k}} \varphi \mathrm{V}(E, A)<3$. 
These conditions are structural restrictions excluding the following subproblems of infinite representation type (see [10]) given by their basic bigraphs from the consideration.

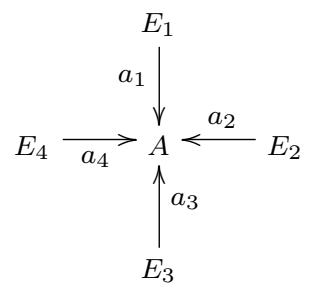

$\mathcal{A}^{1}$

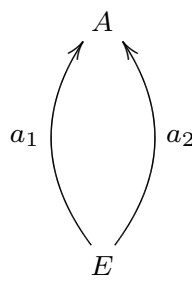

$\mathcal{A}^{2}$

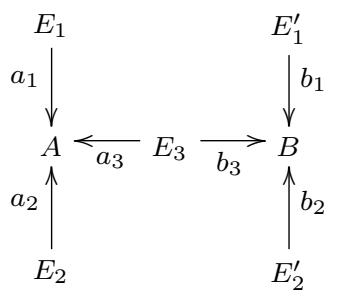

$\mathcal{A}^{3}$

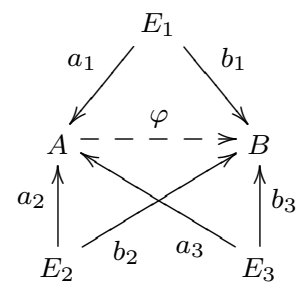

$\mathcal{A}^{4}$

3.4. Quasi multiplicative basis. Let $\mathcal{A} \in \mathrm{C}$. For any $x \in \mathrm{R} \cup \mathrm{V}$ there is a basis decomposition

$$
x=\sum_{y \in \Sigma_{1}} \lambda_{y} y
$$

where almost all $\lambda_{y} \in \mathbf{k}$ are equal to 0 . Denote by $\operatorname{con}_{y} x=\lambda_{y}$ the content of $y$ in $x$. Two nonzero elements $x, y \in \mathrm{R} \cup \mathrm{V}$ are called collinear if $\mathbf{k}^{*} x=\mathbf{k}^{*} y$, where $\mathbf{k}^{*}$ denotes the multiplicative field group. In this case we write $x \| y$.

For vertices $A, B \in \Sigma_{0}^{+}, E \in \Sigma_{0}^{-}$, and solid arrows $a \in \Sigma_{1}^{0}(E, A)$ and $b \in \Sigma_{1}^{0}(E, B)$, let

$$
\begin{aligned}
& \mathrm{S}(a, b)=\left\{\xi \in \Sigma_{1}^{1}(A, B) \mid \operatorname{con}_{b}(\xi a) \neq 0\right\}, \\
& \mathrm{C}(a, b)=\left\{\xi \in \Sigma_{1}^{1}(A, B) \mid \xi a \| b\right\} \subset \mathrm{S}(a, b) .
\end{aligned}
$$

A pair $(a, b)$ is called adjusted if $\mathrm{S}(a, b)=\mathrm{C}(a, b)$. For any $\varphi \in \Sigma_{1}^{1}$, denote

$$
\begin{aligned}
\mathrm{P}_{\varphi}= & \left\{(a, b) \in \Sigma_{1}^{0} \times \Sigma_{1}^{0} \mid \varphi \in \mathrm{S}(a, b)\right\}, \\
& \mathrm{E}_{\varphi}=\left\{s(a),(a, b) \in \mathrm{P}_{\varphi}\right\} .
\end{aligned}
$$

A dotted arrow $\varphi \in \Sigma_{1}^{1}$ is called single provided $\mathrm{P}_{\varphi}=\{(a, b)\}$ and the pair $(a, b)$ is adjusted, and joint if

$$
\mathrm{P}_{\varphi}=\left\{\left(a_{1}, b_{1}\right),\left(a_{2}, b_{2}\right)\right\}
$$

with $a_{1} \neq a_{2}, b_{1} \neq b_{2}$, and the pairs $\left(a_{1}, b_{1}\right),\left(a_{2}, b_{2}\right)$ are adjusted. Obviously, if $\mathcal{A}$ is faithful, $\mathrm{P}_{\varphi} \neq \varnothing$ for any $\varphi \in \Sigma_{1}^{1}$.

Dotted arrows $\varphi_{1}, \varphi_{2} \in \Sigma_{1}^{1}(A, B), A, B \in \Sigma_{0}^{+}, A \neq B$, will be called joint parallel whenever $\operatorname{ord} A=\operatorname{ord} B=3$, and there are $E_{0}, E_{1}, E_{2} \in \Sigma_{0}^{-}$, $a_{i} \in \Sigma_{1}^{0}\left(E_{i}, A\right), b_{i} \in \Sigma_{1}^{0}\left(E_{i}, B\right), i=0,1,2$, such that (see diagram 1 below):

1) $\mathrm{C}\left(a_{0}, b_{0}\right)=\left\{\varphi_{1}, \varphi_{2}\right\}, \mathrm{C}\left(a_{i}, b_{i}\right)=\left\{\varphi_{i}\right\}, i=1,2$;

2) $\mathrm{P}_{\varphi_{i}}=\left\{\left(a_{0}, b_{0}\right),\left(a_{i}, b_{i}\right)\right\}, i=1,2$.

Some of vertices $E_{0}, E_{1}, E_{2}$ may coincide here, but the arrows $a_{0}, a_{1}, a_{2}$, $b_{0}, b_{1}, b_{2}$ are pairwise different. 
Given $a_{1}, \ldots, a_{t} \in \Sigma_{1}$, define

$$
\mathbf{k}^{*}\left\langle a_{1}, \ldots, a_{t}\right\rangle=\left\{\sum_{i=1}^{t} \lambda_{i} a_{i} \mid \lambda_{i} \in \mathbf{k}^{*}\right\} .
$$

We say that the multiplication rule holds on $\mathcal{A}$ if for any $\varphi, \psi \in \Sigma_{1}^{1}$ with $\psi \varphi \neq 0$ one of the following conditions holds:

1) there is $\tau \in \Sigma_{1}^{1}$ such that $\psi \varphi \| \tau$;

2) $\varphi, \psi$ are joint, and there are single $\tau_{1}, \tau_{2} \in \Sigma_{1}^{1}$ such that $\psi \varphi \in \mathbf{k}^{*}\left\langle\tau_{1}, \tau_{2}\right\rangle$, and there are $E_{1}, E_{2} \in \Sigma_{0}^{-}$with, possibly, $E_{1}=E_{2}, A, B, C \in \Sigma_{0}^{+}$, where two of the vertices $A, B, C$ may be equal, and there exist $a_{i} \in \Sigma_{1}^{0}\left(E_{i}, A\right)$, $b_{i} \in \Sigma_{1}^{0}\left(E_{i}, B\right), c_{i} \in \Sigma_{1}^{0}\left(E_{i}, C\right)$ such that $\varphi a_{i}\left\|b_{i}, \psi b_{i}\right\| c_{i}, i=1,2$, and $\tau_{j} a_{i} \| \delta_{i j} c_{i}, i, j=1,2$ where $\delta_{i j}$ is the Kronecker delta (see diagram 2).

Diagr.1

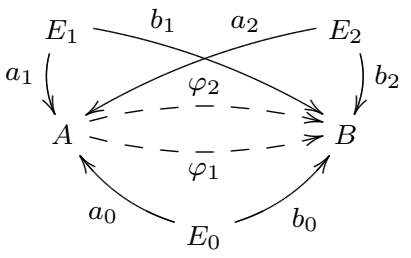

Diagr.2

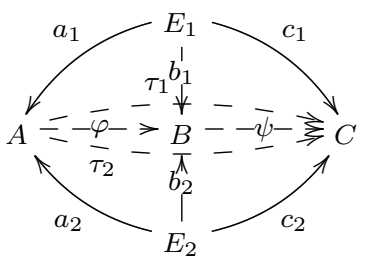

A triangled basis $\Sigma$ of a bimodule problem $\mathcal{A} \in C$ is called quasi multiplicative if the following properties hold:

a) Any pair $(a, b) \in \Sigma_{1}^{0} \times \Sigma_{1}^{0}$ with $\mathrm{S}(a, b) \neq \varnothing$ is adjusted.

b) Any $\varphi \in \Sigma_{1}^{1}$ with $\mathrm{P}_{\varphi} \neq \varnothing$ is either single or joint.

c) For any $a \in \Sigma_{1}^{0}(E, A), b \in \Sigma_{1}^{0}(E, B)$, the inequality $|\mathrm{C}(a, b)| \leqslant 2$ holds. If $\mathrm{C}(a, b)=\left\{\varphi_{1}, \varphi_{2}\right\}$, then $\varphi_{1}, \varphi_{2}$ are joint parallel.

d) The multiplication rule holds on $\mathcal{A}$.

According to $[4$, Theorem 1, p. 8], for a faithful connected finite dimensional bimodule problem $\mathcal{A}$ from class $\mathrm{C}$, there exists a quasi multiplicative basis.

\section{StandARD Minimal NON-SCHURIAN BIMODULE PROBLEM}

4.1. 2-dimensional complex for one-sided bimodule problem. A 2dimensional cell complex $\mathfrak{L}=\left(\mathfrak{L}_{0}, \mathfrak{L}_{1}, \mathfrak{L}_{2}\right)$ associated with a bimodule problem $\mathcal{A} \in \mathcal{C}$ having a quasi multiplicative basic bigraph $\Sigma=\left(\Sigma_{0}, \Sigma_{1}\right)$ consists of:

- the sets $\mathfrak{L}_{0}=\Sigma_{0}, \mathfrak{L}_{1}=\Sigma_{1}$;

- the set $\mathfrak{L}_{2}$ of 2 -cells formed by the set $\mathrm{T}$ of triangles $\Delta=(a, b, \varphi)$ for a $\varphi \in \Sigma_{1}^{1}$ such that $\operatorname{con}_{a}(\varphi b) \neq 0$, and the set $\mathrm{Q}$ of quadrangles $\diamond$ for a 
joint arrow $\varphi \in \Sigma_{1}^{1}$ with $\mathrm{P}_{\varphi}=\left\{\left(a_{1}, b_{1}\right),\left(a_{2}, b_{2}\right)\right\}, a_{1} \neq a_{2}, b_{1} \neq b_{2}$. We can depict quadrangle by the following bigraph:

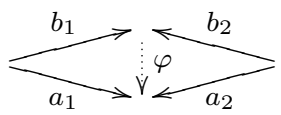

- a map $\partial=\partial_{\mathfrak{L}}: \mathfrak{L}_{2} \rightarrow$ Cycles $_{\Sigma}$ is defined by $\partial(\triangle)=\left\langle\varphi b a^{-1}\right\rangle$ for a triangle $\triangle$, and by $\partial(\diamond)=\left\langle a_{2}^{-1} a_{1} b_{1}^{-1} b_{2}\right\rangle$ for a quadrangle $\diamond$.

Remark 4.2. Definition of a quasi multiplicative basis for a bimodule problem $\mathcal{A} \in \mathcal{C}$ implies the following cell properties:

1) every $\varphi \in \Sigma_{1}^{1}$ with $\mathrm{P}_{\varphi} \neq \varnothing$ belongs either to one or two triangles from the set

$$
\mathrm{T}_{0}=\left\{(a, b, \varphi) \in \mathrm{T} \mid a, b \in \Sigma_{1}^{0}\right\} ;
$$

2) for $a, b \in \Sigma_{1}^{0}, a \neq b$, such that $s(a)=s(b)$, the pair $(a, b)$ belongs to two triangles from $\mathrm{T}_{0}$ at most;

$3)$ if $\left(a_{1}, b_{1}, \varphi\right),\left(a_{2}, b_{2}, \varphi\right) \in \mathrm{T}_{0}$ are different triangles, then $a_{1} \neq a_{2}, b_{1} \neq b_{2}$;

$4)$ if $\left(a, b, \varphi_{1}\right),\left(a, b, \varphi_{2}\right) \in \mathrm{T}_{0}$ and $\varphi_{1} \neq \varphi_{2}$, then there exist $\left(a_{i}, b_{i}, \varphi_{i}\right) \in \mathrm{T}_{0}$, $i=1,2$, such that $a_{1} \neq a_{2}, b_{1} \neq b_{2}$.

\subsection{Universal covering associated with a quasi multiplicative basis of schurian bimodule problem.}

Lemma 4.4. Let $\mathcal{A} \in \mathcal{C}$ be a connected locally finite dimensional bimodule problem, let $\mathfrak{L}=\mathfrak{L}_{\mathcal{A}}$ be 2-dimensional cell complex associated with a quasi multiplicative basis $\Sigma=\Sigma_{\mathcal{A}}$ of $\mathcal{A}$, and let $\mathrm{p}_{\mathfrak{L}}: \widetilde{\mathfrak{L}} \rightarrow \mathfrak{L}$ be a covering of abstract complexes. Then there exist a locally finite dimensional bimodule problem $\tilde{\mathcal{A}}=(\tilde{\mathrm{K}}, \tilde{\mathrm{V}}) \in \mathcal{C}$ with a basis $\tilde{\Sigma}$ and associated 2-dimensional cell complex $\tilde{\mathfrak{L}}$, and a Galois covering morphism $\mathrm{p}_{\mathcal{A}}: \tilde{\mathcal{A}} \rightarrow \mathcal{A}$ with the fundamental group $G(\mathfrak{L})$ such that the diagram

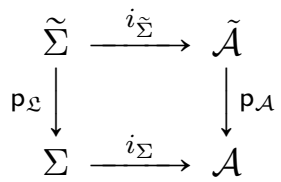

commutes, where $i_{\Sigma}: \Sigma \rightarrow \mathcal{A}$ and $i_{\widetilde{\Sigma}}: \widetilde{\Sigma} \rightarrow \mathcal{A}$ are natural embeddings.

Proof. Let $\mathrm{Ob} \tilde{\mathrm{K}}=\widetilde{\mathfrak{L}}_{0}$, and let the spaces $\operatorname{Rad} \tilde{\mathrm{K}}(\tilde{X}, \tilde{Y}), \tilde{\mathrm{V}}(\tilde{X}, \tilde{Y})$ be freely generated over $\mathbf{k}$ by $\widetilde{\mathfrak{L}}_{1}^{1}(\tilde{X}, \tilde{Y})$ and $\widetilde{\mathfrak{L}}_{1}^{0}(\tilde{X}, \tilde{Y})$ correspondingly, $\tilde{X}, \tilde{Y} \in$ Ob $\tilde{\mathrm{K}}$. If $\tilde{a}: \tilde{X} \rightarrow \tilde{Y}, \tilde{b}: \tilde{Y} \rightarrow \tilde{Z}$ are two elements of $\tilde{\mathfrak{L}}_{1}, \mathrm{p}_{1}(\tilde{a})=a, \mathrm{p}_{1}(\tilde{b})=b$, then for any $x \in \mathfrak{L}_{1}$ such that $\operatorname{con}_{x}(b a) \neq 0$ and for a unique $\tilde{x} \in \widetilde{\mathfrak{L}}_{1}$ such that $\mathrm{p}_{1}(\tilde{x})=x, s(\tilde{x})=s(\tilde{a})$, we set $\operatorname{con}_{\tilde{x}}(\tilde{b} \tilde{a})=\operatorname{con}_{x}(b a)$ in $\tilde{\mathcal{A}}$, and $\operatorname{con}_{\tilde{y}}(\tilde{b} \tilde{a})=0$ 
for any other $\tilde{y} \in \widetilde{\mathfrak{L}}_{1}$. The composition $\tilde{b} \tilde{a}$ is correctly defined since $\left\langle\tilde{b} \tilde{a} \tilde{x}^{-1}\right\rangle$ is a bound of a cell in $\tilde{\mathfrak{L}}$, and hence it is a cycle in $\tilde{\mathfrak{L}}$. Associativity of such composition is obvious. $\tilde{\mathrm{K}}$-bimodule structure on $\tilde{\mathrm{V}}$ is defined similarly. The covering morphism $\mathrm{p}_{\mathcal{A}}: \tilde{\mathcal{A}} \rightarrow \mathcal{A}$ is uniquely defined by the commutativity of the diagram.

Remark 4.5. Let $\mathrm{p}_{\mathcal{A}}: \tilde{\mathcal{A}} \rightarrow \mathcal{A}$ be a Galois covering morphism of bimodule problems, and let $\Sigma$ be a basis of $\mathcal{A}$. Then inverse images of elements of $\Sigma_{1}$ form a basis $\tilde{\Sigma}$ of $\tilde{\mathcal{A}}$, and $\mathrm{p}_{\mathcal{A}}$ induces the associated covering $\mathrm{p}_{\mathfrak{L}}: \tilde{\mathfrak{L}} \rightarrow \mathfrak{L}$ of complexes. This construction is inverse to one in Lemma 4.4.

For a bimodule problem $\mathcal{A} \in \mathcal{C}$, let $\mathrm{p}: \widetilde{\mathfrak{L}} \rightarrow \mathfrak{L}$ be the constructed above universal covering of 2-dimensional complex $\mathfrak{L}=\mathfrak{L}_{\mathcal{A}}$. By Lemma 4.4, there exists a (corresponding to $\mathrm{p}$ ) covering morphism $\mathrm{p}_{\mathcal{A}}: \tilde{\mathcal{A}} \rightarrow \mathcal{A}$ of bimodule problems which we will call a universal covering of bimodule problem $\mathcal{A}$ associated with the complex $\mathfrak{L}$.

A bimodule problem $\mathcal{A}$ is said to be simply connected provided 2-dimensional complex $\mathfrak{L}$ over $\Sigma$ is connected and its fundamental group $G(\mathfrak{L})$ is trivial. Obviously, simply connected schurian bimodule problem $\mathcal{A}$ is isomorphic to its universal covering $\tilde{\mathcal{A}}$.

4.6. Minimal non-schurian bimodule problem. Recall that for a sincere schurian bimodule problem $\mathcal{A}$, its basis $\Sigma_{\mathcal{A}}$ is 0-connected. If bimodule problem $\mathcal{A}$ is non-faithful, then every sincere $M \in \operatorname{ind} \mathcal{A}$ is non-schurian. Therefore, each sincere schurian bimodule problem is faithful.

Let $\mathcal{A}=(\mathrm{K}, \mathrm{V})$ be a sincere non-schurian bimodule problem such that Tits form $q_{\mathcal{A}} \in \mathrm{WP}$, and for every proper subset $S \subset \mathrm{ObK}$ the restricted bimodule problem $\mathcal{A}_{S}$ is schurian. Then $\mathcal{A}$ is called a minimal non-schurian bimodule problem.

Let $\mathcal{B}, \mathcal{C}$ be bimodule problems defined by their bigraphs: $\left(\Sigma_{\mathcal{B}}\right)_{0}=\{X\}$, $\left(\Sigma_{\mathcal{B}}\right)_{1}=\varnothing,\left(\Sigma_{\mathcal{C}}\right)_{0}=\{X, Y\},\left(\Sigma_{\mathcal{C}}\right)_{1}=\left(\Sigma_{\mathcal{C}}\right)_{1}^{0}=\{a: X \rightarrow Y\}$. If $\mathcal{A} \in \mathcal{C}$, $\left|\left(\Sigma_{\mathcal{A}}\right)_{0}\right| \leqslant 2$, then $\mathcal{A}$ is sincere schurian if and only if $\mathcal{A} \in\{\mathcal{B}, \mathcal{C}\}$.

This observation excludes the non-schurian problems having at most two vertices from the consideration, and helps to describe the minimal nonschurian bimodule problems containing at least 3 vertices.

Lemma 4.6.1 $([3,6])$. Let $\mathcal{A}=(\mathrm{K}, \mathrm{V})$ be a minimal non-schurian admitted bimodule problem with a basis $\Sigma$, and $\left|\Sigma_{0}\right| \geqslant 3$. If $\mathcal{A}_{\mathrm{red}}=\left(\mathrm{K} / \mathrm{Ann}_{\mathrm{K}} \mathrm{V}, \mathrm{V}\right)$ is a sincere schurian bimodule problem, then there exist two uniquely defined vertices $A, B \in \Sigma_{0}^{+}$such that:

1) for any sincere $M \in \operatorname{ind} \mathcal{A}$, the vector $\operatorname{dim}_{\mathcal{A}} M$ is a basic root of Tits quadratic form $q_{\mathcal{A}_{\mathrm{red}}}$ with the singular vertices $A, B$; 
2) if $\mathrm{Ann}_{\mathrm{K}} \mathrm{V}\left(A_{1}, B_{1}\right) \neq 0$, then the sets $\left\{A_{1}, B_{1}\right\},\{A, B\}$ coincide.

A minimal non-schurian bimodule problem $\mathcal{A}$ satisfying the conditions of Lemma 4.6.1 is called a standard minimal non-schurian bimodule problem with singular vertices $A$ and $B$.

4.7. Schurity and coverings: the main result. By the construction of bimodule problem universal covering $\mathrm{p}: \tilde{\mathcal{A}} \rightarrow \mathcal{A}$, we assume that

$$
\mathrm{p}_{i}\left(\widetilde{\Sigma}_{i}\right)=\Sigma_{i}, \quad i=0,1,
$$

for the bases $\widetilde{\Sigma}$ and $\Sigma$ of $\tilde{\mathcal{A}}$ and $\mathcal{A}$ respectively.

Theorem 4.7.1. Let $\mathcal{A} \in \mathcal{C}$ be a connected finite dimensional bimodule problem with weakly positive Tits form $q_{\mathcal{A}}$, let $\tilde{\mathcal{A}} \in \mathcal{C}$ be a schurian bimodule problem, and let $\mathrm{p}: \tilde{\mathcal{A}} \rightarrow \mathcal{A}$ be a universal covering. Then either $\mathcal{A}$ is schurian, or contains a dotted loop, or some restriction $\mathcal{A}_{S}$ is a standard minimal non-schurian bimodule problem.

Proof. Suppose that $\mathcal{A}=(\mathrm{K}, \mathrm{V})$ is not schurian and does not contain dotted loops. Let $\Sigma$ and $\tilde{\Sigma}$ be the bases of $\mathcal{A}$ and $\tilde{\mathcal{A}}$ correspondingly such that $\mathrm{p}(\tilde{\Sigma})=\Sigma$. We will mark the object (vertex, arrow, etc.) related to the covering $\tilde{\mathcal{A}}$ by the sign $\sim$, and its image in $\mathcal{A}$ will be denoted by the same letter without .

Since the bimodule problem $\tilde{\mathcal{A}}$ is schurian and $\mathcal{A}$ is not, there exists a representation $\tilde{X} \in$ ind $\tilde{\mathcal{A}}$ such that for $\tilde{S}=\operatorname{supp} \tilde{X}$, the induced morphism $\mathrm{p}_{\tilde{S}}: \tilde{\mathcal{A}}_{\tilde{S}} \rightarrow \mathcal{A}_{S}$ is not an isomorphism. Then the restriction $\mathcal{A}_{S}$ is sincere minimal non-schurian. Let us choose $\tilde{X}$ with the minimal possible $|\tilde{S}|$.

Note that for any $S \subset \Sigma_{0},|S| \leqslant 2$, the restriction $\mathcal{A}_{S}$ is schurian by definition of class $C$ and our assumption. Therefore one can assume $|S| \geqslant 3$, and hence $|\tilde{S}| \geqslant 3$. By Remark 4.5 , the morphism $\mathrm{p}_{\tilde{S}}: \widetilde{\mathfrak{L}}_{\tilde{S}} \rightarrow \mathfrak{L}_{S}$ of the associated complexes induced by $\mathrm{p}$ is not an isomorphism. Therefore:

1) either the map $\left.\mathrm{p}_{0}\right|_{\tilde{S}}:\left(\widetilde{\Sigma}_{\tilde{S}}\right)_{0} \rightarrow\left(\Sigma_{S}\right)_{0}$ induced by $\mathrm{p}$ is not a bijection on the vertices;

2) or the map $\left.\mathrm{p}_{0}\right|_{\tilde{S}}$ is a bijection, but there exist $\tilde{A}_{1}, \tilde{A}_{2} \in \tilde{S}$ such that

$$
\mathrm{p}\left(\widetilde{\Sigma}_{1}\left(\tilde{A}_{1}, \tilde{A}_{2}\right)\right) \neq \Sigma_{1}\left(A_{1}, A_{2}\right) .
$$

Step 1. The first case is impossible.

Proof. Suppose there exist $\tilde{A}_{1}, \tilde{A}_{2} \in \tilde{S}, \tilde{A}_{1} \neq \tilde{A}_{2}$, with $A_{1}=A_{2} \in S$. Since $\tilde{\mathcal{A}}_{\tilde{S}}$ is schurian, by Lemma $2.6, \tilde{x}=\operatorname{dim} \tilde{X}$ is a sincere positive root of weakly positive unit quadratic form $q_{\tilde{\mathcal{A}}_{\tilde{S}}}$. If $\tilde{x}$ is not basic with singular vertices $\tilde{A}_{1}, \tilde{A}_{2}$, then, by Lemma 1.3.1 there is a non-sincere $\tilde{y}<\tilde{x}$ such that $\tilde{A}_{1}, \tilde{A}_{2} \in \operatorname{supp} \tilde{y}$ which contradicts to the minimality of $|\tilde{S}|$ by Lemma 2.6. 
Therefore, $\tilde{x}$ is a basic root with the singular vertices $\tilde{A}_{1}, \tilde{A}_{2}$. By definition of a basic root, the vertices $\tilde{A}_{1}$ and $\tilde{A}_{2}$ are defined uniquely, i.e. $B_{1} \neq B_{2}$ for any pair $\tilde{B}_{1}, \tilde{B}_{2} \in \tilde{S}$ of different vertices such that

$$
\left\{\tilde{B}_{1}, \tilde{B}_{2}\right\} \neq\left\{\tilde{A}_{1}, \tilde{A}_{2}\right\} .
$$

Since $\tilde{\mathcal{A}}_{\tilde{S}}$ is 0 -connected, there is a vertex $\tilde{C} \in \tilde{S}$ connected with $\tilde{A}_{1}$ by a solid arrow $\tilde{a}: \tilde{C} \rightarrow \tilde{A}_{1}$ (up to direction of $\tilde{a}$ ). If $\tilde{C}=\tilde{A}_{2}$, then the quadratic form $q_{\tilde{\mathcal{A}}_{\tilde{S}}}$ is not WP. So $\tilde{C} \neq \tilde{A}_{2}$. By Lemma 2.6, for a positive root $\tilde{z}=\tilde{x}-e_{\tilde{A}_{1}}$, there is an indecomposable representation $\tilde{Z}$ of the dimension $\tilde{z}$ with $|\operatorname{supp} \tilde{Z}|<|\tilde{S}|$. The corresponding restriction

$$
\mathrm{p}_{\text {supp } \tilde{Z}}: \tilde{\mathcal{A}}_{\text {supp } \tilde{Z}} \rightarrow \mathcal{A}_{S}
$$

is not an isomorphism since the arrow $a: C \rightarrow A_{1}$ does not have an inverse image in $\tilde{\Sigma}_{1}\left(\tilde{C}, \tilde{A}_{2}\right)$, which contradicts to the minimality of $|\tilde{S}|$.

Now we can assume that the restriction $\left.\mathrm{p}_{0}\right|_{\tilde{S}}$ is a bijection, and the case 2) holds.

Step 2. Let $\tilde{A}_{1}, \tilde{A}_{2} \in \tilde{S}$ and $\mathrm{p}\left(\widetilde{\Sigma}_{1}\left(\tilde{A}_{1}, \tilde{A}_{2}\right)\right) \neq \Sigma_{1}\left(A_{1}, A_{2}\right)$. Then the set of vertices $\left\{\tilde{A}_{1}, \tilde{A}_{2}\right\}$ is uniquely defined, and $\operatorname{dim}_{\tilde{\mathcal{A}}} \tilde{X}$ is a basic root of $q_{\tilde{\mathcal{A}}_{\tilde{S}}}$ with singular vertices $\tilde{A}_{1}, \tilde{A}_{2}$.

The proof is similar.

Step 3. The set

$$
\mathcal{X}=\left(\Sigma _ { 1 } ( A _ { 1 } , A _ { 2 } ) \backslash \mathrm { p } _ { 1 } ( \widetilde { \Sigma } _ { 1 } ( \tilde { A } _ { 1 } , \tilde { A } _ { 2 } ) ) \cup \left(\Sigma_{1}\left(A_{2}, A_{1}\right) \backslash \mathrm{p}_{1}\left(\widetilde{\Sigma}_{1}\left(\tilde{A}_{2}, \tilde{A}_{1}\right)\right)\right.\right.
$$

consists of dotted arrows.

Proof. Since bimodule problem $\mathcal{A}$ is admitted, either $\mathcal{X} \subset \Sigma_{1}^{0}$, or $\mathcal{X} \subset \Sigma_{1}^{1}$. Suppose that $\mathcal{X} \subset \Sigma_{1}^{0}$. Let

$$
x=\operatorname{dim}_{\mathcal{A}} \operatorname{rep} \mathrm{p}(\tilde{X}) .
$$

Then $x_{A}=\tilde{x}_{\tilde{A}}$ for any $\tilde{A} \in \tilde{S}$. Hence

$$
\begin{aligned}
q_{\mathcal{A}}(x) & =\sum_{(A, B) \in S \times S}\left(\delta_{A B}+\left|\Sigma_{1}^{1}(A, B)\right|-\left|\Sigma_{1}^{0}(A, B)\right|\right) x_{A} x_{B}= \\
& =\sum_{(\tilde{A}, \tilde{B}) \in \tilde{S}}\left(\delta_{\tilde{A} \tilde{B}}+\left|\widetilde{\Sigma}_{1}^{0}(\tilde{A}, \tilde{B})\right|-\left|\widetilde{\Sigma}_{1}^{0}(\tilde{A}, \tilde{B})\right|\right) x_{\tilde{A}} x_{\tilde{B}}-\sum_{a \in \mathcal{X}} x_{A_{1}} x_{A_{2}}= \\
& =q_{\tilde{\mathcal{A}}}(\tilde{x})-\sum_{a \in \mathcal{X}} x_{A_{1}} x_{A_{2}}=1-\sum_{a \in \mathcal{X}} x_{A_{1}} x_{A_{2}} \leqslant 1-1=0,
\end{aligned}
$$

that contradicts to the weak positivity of $q_{\mathcal{A}}$. 
Step 4. $\mathcal{X} \subset \mathrm{Ann}_{\mathrm{K}_{S}} \mathrm{~V}_{S}$.

Proof. If $\varphi \in \mathcal{X} \backslash \mathrm{Ann}_{\mathrm{K}_{S}} \mathrm{~V}_{S}$, then (up to direction of $\varphi$ ) there is a triangle $\left(a_{1}, a_{2}, \varphi\right) \in \mathrm{T}_{0}$ with $a_{1}: B \rightarrow A_{1}, a_{2}: B \rightarrow A_{2}$ for some $B \in S$. Since the triangles are lifted up in the covering, then for the unique $\tilde{B} \in \tilde{S}$ there exist $\tilde{a}_{1}: \tilde{B} \rightarrow \tilde{A}_{1}, \tilde{a}_{2}: \tilde{B} \rightarrow \tilde{A}_{2}$ in $\widetilde{\Sigma}$ and a triangle $\left(\tilde{a}_{1}, \tilde{a}_{2}, \tilde{\varphi}\right) \in \tilde{T}_{0}$. Therefore $\mathrm{p}_{0}(\tilde{\varphi})=\varphi$ that contradicts to the definition of $\mathcal{X}$.

Hence,

$$
\left(\mathcal{A}_{S}\right)_{\mathrm{red}}=\left(\mathrm{K}_{S} / \mathrm{Ann}_{\mathrm{K}_{S}} \mathrm{~V}_{S}, \mathrm{~V}_{S}\right)
$$

is isomorphic to $\tilde{\mathcal{A}}_{\tilde{S}}$, and therefore $\left(\mathcal{A}_{S}\right)_{\text {red }}$ is sincere schurian. Then due to Lemma 4.6.1, $\mathcal{A}_{S}$ is standard minimal non-schurian bimodule problem, which completes the proof of Theorem 4.7.1.

\section{CONCLUSION}

The article is a part of research of the representation finiteness problem for a wide class of multi-vector space categories consisting of so called onesided bimodule problems. We use the construction of a universal covering of an one-sided bimodule problem in order to obtain some schurity criterium for a bimodule problem from our class. We are going to study representation type of one-sided bimodule problems using developed technique.

\section{REFERENCES}

[1] V. M. Babych, N. S. Golovashchuk. An application of covering techniques. Nauk. Vīsn. Uzhgorod. Univ. Ser. Mat. Inform., 8:4-14, 2003.

[2] V. M. Babych, N. S. Golovashchuk. Bimodule problems and cell complexes. Algebra Discrete Math., 3:17-29, 2006.

[3] V. M. Babych, N. S. Golovashchuk. On schurity of one-sided bimodule problems. Algebra and Discrete Mathematics, 28(2):157-170, 2019.

[4] V. M. Babych, N. S. Golovashchuk, S. A. Ovsienko. Generalized multiplicative bases for one-sided bimodule problems. Algebra Discrete Math., 12(2):1-24, 2011.

[5] F. Bogomolov, Yu. Tschinkel, editors. Geometric methods in algebra and number theory, volume 235 of Progress in Mathematics. Birkhäuser Boston, Inc., Boston, MA, 2005, doi: $10.1007 / \mathrm{b} 138649$. Including papers from the Winter School held at the University of Miami, Miami, FL, December 16-20, 2003.

[6] V. M. Bondarenko, N. S. Golovashuk, S. A. Ovsienko, A. V. Roiter. Schurian matrix problems and quadratic forms. Inst. of Math., Academy of Sci. USSR. Preprint 78.25, pages 19-38, 1978 [in Russian].

[7] K. Bongartz. Critical simply connected algebras. Manuscripta Math., 46(1-3):117-136, 1984, doi: 10.1007/BF01185198.

[8] K. Bongartz, P. Gabriel. Covering spaces in representation-theory. Invent. Math., 65(3):331-378, 1981/82, doi: 10.1007/BF01396624.

[9] A. C. Da Silva, A. Weinstein. Geometric Models for Noncommutative Algebras, volume 10 of Berkeley mathematics lecture notes. American Mathematical Soc., 1999. 
[10] Yu. A. Drozd. Tame and wild matrix problems. In: Representations and quadratic forms, Kiev:39-74, 1979 [English translation in AMS Translations v. 128].

[11] Yu. A. Drozd, S. A. Ovsienko. Coverings of tame boxes and algebras. Preprint MPI 00-26, Max-Plank-Institut für Mathematik, Bonn, 2000.

[12] Yu. A. Drozd, S. A. Ovsienko, B. Yu. Furchin. Categorical constructions in representations theory. In: Algebraic Structures and Their Applications, Kiev State Univ., Kiev:17-43, 1988.

[13] P. Gabriel. Indecomposable representations. II. In Symposia Mathematica, Vol. XI (Convegno di Algebra Commutativa, INDAM, Rome, 1971), pages 81-104. 1973.

[14] P. Gabriel. Auslander-Reiten sequences and representation-finite algebras. In Representation theory, I (Proc. Workshop, Carleton Univ., Ottawa, Ont., 1979), volume 831 of Lecture Notes in Math., pages 1-71. Springer, Berlin, 1980.

[15] P. Gabriel, A. V. Roiter. Representations of finite-dimensional algebras. In Algebra, VIII, volume 73 of Encyclopaedia Math. Sci., pages 1-177. Springer, Berlin, 1992. With a chapter by B. Keller.

[16] O. T. Izhboldin, B. Kahn, N. A. Karpenko, A.r Vishik. Geometric methods in the algebraic theory of quadratic forms, volume 1835 of Lecture Notes in Mathematics. Springer-Verlag, Berlin, 2004, doi: 10.1007/b94827. Edited by Jean-Pierre Tignol.

[17] M. M. Kleiner, A. V. Roiter. Representations of differential graded categories. In Representations of algebras (Proc. Internat. Conf., Carleton Univ., Ottawa, Ont., 1974), pages 316-339. Lecture Notes in Math., Vol. 488. 1975.

[18] A. Ol'shanskii. The Geometry of Defining Relations in Groups. Kluwer Academic Publishers, 1991.

[19] S. A. Ovsienko. Bimodule and matrix problems. Progress in Mathematics, 173:323-357, 1999.

[20] C. M. Ringel. Tame algebras and integral quadratic forms, volume 1099 of Lecture Notes in Mathematics. Springer-Verlag, Berlin, 1984, doi: 10.1007/BFb0072870.

[21] A. V. Roiter, V. V. Sergejchuk. Existence of a multiplicative basis for a finitely spaced module over an aggregate. Ukrainian Math. Journal, 46(5):567-579 (Engl. transl. 604617), 1994.

[22] G. W. Whitehead. Elements of homotopy theory, volume 61 of Graduate Texts in Mathematics. Springer-Verlag, New York-Berlin, 1978.

Received: June 26, 2020, accepted: June 12, 2021.

Vyacheslav Babych

Department of Mechanics and Mathematics, Taras Shevchenko National UniVERSity of Kyiv, 64, Volodymyrs'Ka St., Kyiv, Ukraine

Email: vyacheslav.babych@univ.kiev.ua

Nataliya Golovashchuk

Department of Mechanics and Mathematics, Taras Shevchenko National UniVERSity of Kyiv, 64, Volodymyrs'Ka St., Kyiv, Ukraine

Email: golova@univ.kiev.ua 